\title{
Proof theory for quantified monotone modal logics
}

\section{Negri, Sara}

2019-08

Negri , S \& Orlandelli , E 2019 , ' Proof theory for quantified monotone modal logics ' , Logic pÿjournal of the IGPL , vol. 27 , no. 4 , pp. 478506 . https://doi.org/10.1093/jigpal/jzz015

http://hdl.handle.net/10138/307488

https://doi.org/10.1093/jigpal/jzz015

gnu_lgpl

acceptedVersion

Downloaded from Helda, University of Helsinki institutional repository.

This is an electronic reprint of the original article.

This reprint may differ from the original in pagination and typographic detail.

Please cite the original version. 


\title{
Proof theory for quantified monotone modal logics
}

\author{
Sara Negri, Department of Philosophy, University of Helsinki, \\ Unioninkatu 40A, Helsinki, Finland. Email: sara.negri@helsinki.fi \\ Eugenio Orlandelli, Department of Philosophy and Communication \\ Studies, University of Bologna, via Zamboni 38, Bologna, Italy. \\ Email: eugenio.orlandelli@unibo.it
}

\begin{abstract}
This paper provides a proof-theoretic study of quantified non-normal modal logics. It introduces labelled sequent calculi based on neighbourhood semantics for the first-order extension, with both varying and constant domains, of monotone non-normal modal logics, and studies the role of the Barcan Formulas in these calculi. It will be shown that the calculi introduced have good structural properties: invertibility of the rules, height-preserving admissibility of weakening and contraction, and syntactic cut elimination. It will also be shown that each of the calculi introduced is sound and complete with respect to the appropriate class of neighbourhood frames. In particular, the completeness proof constructs a formal derivation for derivable sequents and a countermodel for underivable ones, and gives a semantic proof of the admissibility of cut.
\end{abstract}

Keywords: Non-normal modal logics, quantified modal logics, labelled sequent calculus, neighbourhood semantics, Barcan Formulas.

\section{Introduction}

Propositional non-normal modal logics allow us to deal with many interpretations of modal operators in which the schema $\square(A \wedge B) \supset(\square A \wedge \square B)$ and/or the necessitation rule $\vdash A / \vdash \square A$ don't seem to hold - e.g., with epistemic [19], game-theoretic [18], and 'high-probability' interpretations [10]. Quantified non-normal modal logics have been studied in $[1,2,3,4,20,21]$. In particular, [1, 2, 21] study constant domain neighbourhood semantics, give characterization results for the Barcan Formulas, and consider axiomatic calculi for non-normal logics; see [2] for the main results. Varying domain semantics for non-normal logics and axiomatic systems for them are studied in $[4,20]$ (and in the unpublished [3]). Nevertheless, [20] characterizes the Barcan Formulas in terms of neighbourhood models instead of frames, and [4] considers multirelational semantics instead of the more general neighbourhood semantics. Even if we focus our attention only on quantified monotone modal logics (QMML), that is logics where the schema $\square(A \wedge B) \supset(\square A \wedge \square B)$ holds, we have interesting applications. To illustrate, [2] points that under a 'high-probability' interpretation of the modal operator $\square$, the Barcan Formula $B F \equiv \forall x \square A \supset \square \forall x A$ would be responsible for the lottery paradox: one instance of $B F$ would say that 'if each individual ticket of a lottery probably is a loser then probably all tickets are losers' [2, p.176].

One limitation of these works on quantified non-normal modal logics is that com- 


\section{Proof theory for quantified monotone modal logics}

pleteness results of axiomatic systems with respect to classes of frames are not modular and in many cases axiomatic systems are incomplete.

In recent years, the development of analytic sequent calculi for propositional nonnormal modal logics has been the object of active investigation, see $[8,13,17]$ and the related [9] where analytic labelled tableaux are introduced. In particular, [13] extends the applicability of labelled sequent calculi [12] to propositional non-normal modal logics through the internalization of neighbourhood semantics. We extend this approach to the first-order case by internalizing the semantics of quantified monotone neighbourhood frames. We shall start from neighbourhood frames with both varying and constant domains, and then analyze the Barcan Formulas. As main results, we will show that each labelled calculus considered has good structural properties and is sound and complete with respect to the appropriate class of neighbourhood frames. As for quantified normal modal logics, labelled calculi allow for an elegant and uniform proof of completeness for all the QMML considered. This is a strong motivation for the present approach. The case of quantified non-monotone logics is left for future research.

The paper is organized as follows: Section 2 sketches propositional non-normal modal logics based on neighbourhood semantics and it shows how the semantics can be simplified for monotonic logics. Then, it presents the propositional labelled calculi based on monotone neighbourhood frames introduced in [13]. Section 3 introduces labelled calculi for QMML without the Barcan Formulas. The calculi considered are based on neighbourhood frames with either varying or constant domains. Then, the structural properties of these calculi are studied. The main results will be that all rules are height-preserving invertible, that the rules of weakening and contraction are height-preserving admissible, and that cut is admissible. Section 4 proves that each calculus considered is sound and complete with respect to the appropriate class of neighbourhood frames. Section 5 considers the Necessity of Identity and the Barcan Formulas; geometric rules that allow to derive each of these schemata while preserving the good structural properties of the underlying calculi are introduced. Furthermore, the soundness and completeness results proved in Section 3 are extended to the new cases. Lastly, Section 6 discusses other works on quantified non-normal modal logics.

\section{Propositional non-normal modal logics}

\subsection{Language}

Given a (finite or countable) set $\Phi$ of propositional variables, the language of propositional modal logics $\left(\mathcal{L}^{\Phi}\right)$ is generated by the following grammar:

$$
A::=P_{i}|\perp| A \vee A|A \wedge A| A \supset A|\square A| \diamond A ;
$$

where $P_{i} \in \Phi$. We assume the usual conventions for parentheses, and use the following abbreviations: $\neg A \equiv B \supset \perp$ and $A \supset A \equiv(A \supset B) \wedge(B \supset A)$.

The simplest way of introducing propositional non-normal modal logics (NNML) is by presenting them as sets of $\mathcal{L}^{\Phi}$-formulas. We do so, mostly following the naming conventions introduced in [5]. The minimal NNML $\mathbf{E}$ is the smallest set of $\mathcal{L}^{\Phi}$ formulas containing all $\mathcal{L}^{\Phi}$-instances of propositional tautologies and of the schema:

$$
E:=\square A \supset \neg \diamond \neg A,
$$




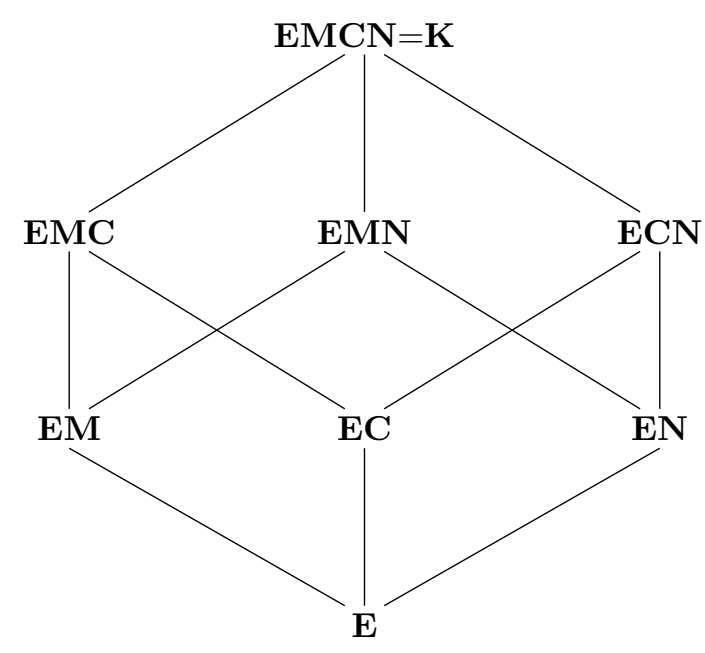

FIG. 1: Lattice of NNML

that is closed under the following rules of inference:

$$
\frac{A \supset B \quad A}{B} M P \quad \frac{A \supset B}{\square A \supset \square B} R E .
$$

Other NNML are obtained by extending $\mathbf{E}$ with some combination of the following axioms:

$$
M:=\square(A \wedge B) \supset(\square A \wedge \square B) \quad C:=(\square A \wedge \square B) \supset \square(A \wedge B) \quad N:=\square \top .
$$

For example, EM (or simply $\mathbf{M}$ ) is the smallest extension of $\mathbf{E}$ containing all $\mathcal{L}^{\Phi}$ instances of the schema $M$. In Figure 1, we have depicted the logical relations among members of NNML. Note that the logic EMCN is equivalent to the minimal normal modal logic K. In this paper we will consider only the first-order extensions of logics above the minimal monotone logic $\mathbf{M}$. If $\mathbf{L}$ is one of these logics, we will sometimes use $\vdash_{\mathbf{L}} A$ to say that $A \in \mathbf{L}$.

\subsection{Neighbourhood semantics for NNML}

DeFinition 2.1 (Frame)

A (neighbourhood) frame is a pair $\mathcal{F} \equiv<W, \mathcal{N}>$, where $W$ is a non-empty set of states (worlds) and $\mathcal{N}$ is a neighbourhood function defined over $W$ :

$$
\mathcal{N}: W \longrightarrow 2^{2^{W}}
$$

DEFINITION 2.2 (Model)

Given a frame $\langle W, \mathcal{N}\rangle$, a (neighbourhood) model defined over it is a triple $\mathcal{M}=<$ $W, \mathcal{N}, \mathcal{V}>$, where $\mathcal{V}$ is a valuation function defined over $\Phi$ :

$$
\mathcal{V}: \Phi \longrightarrow 2^{W} \text {. }
$$


4 Proof theory for quantified monotone modal logics

Definition 2.3 (Truth)

Given a model $\mathcal{M}=\left\langle W, \mathcal{N}, \mathcal{V}>\right.$ and a state $w \in W$, truth of an $\mathcal{L}^{\Phi}$-formula $A$ is recursively defined as follows:

$$
\begin{array}{lll}
\mathcal{M}, w \Vdash P_{i} & \text { iff } & w \in \mathcal{V}\left(P_{i}\right) \\
\mathcal{M}, w \Vdash \perp & & \\
\mathcal{M}, w \Vdash B \vee C & \text { iff } & \mathcal{M}, w \Vdash B \text { or } \mathcal{M}, w \Vdash C \\
\mathcal{M}, w \Vdash B \wedge C & \text { iff } & \mathcal{M}, w \Vdash B \text { and } \mathcal{M}, w \Vdash C \\
\mathcal{M}, w \Vdash B \supset C & \text { iff } & \mathcal{M}, w \Vdash B \text { or } \mathcal{M}, w \Vdash C \\
\mathcal{M}, w \Vdash \square B & \text { iff } & \llbracket B \rrbracket_{\mathcal{M}} \in \mathcal{N}(w) \\
\mathcal{M}, w \Vdash \diamond B & \text { iff } & \llbracket \neg B \rrbracket_{\mathcal{M}} \notin \mathcal{N}(w)
\end{array}
$$

where $P_{i} \in \Phi$ and $\llbracket A \rrbracket_{\mathcal{M}}$ is the truth set of $A$, i.e., $\llbracket A \rrbracket_{\mathcal{M}}=\{v \in W \mid \mathcal{M}, v \Vdash A\}$.

We shall use $w \Vdash A$ in place of $\mathcal{M}, w \Vdash A$ whenever possible; as in [13], $a \Vdash^{\forall} A$ is a shorthand for $\forall v \in W(v \in a \supset v \Vdash A)$, and $a \Vdash^{\exists} A$ for $\exists v \in W(v \in a \wedge v \Vdash A)$.

We say that an $\mathcal{L}^{\Phi}$-formula $A$ is globally true in $\mathcal{M}$ whenever $\llbracket A \rrbracket_{\mathcal{M}}=W$. A formula is valid in a frame $\mathcal{F}$ if it is globally true in all models based on $\mathcal{F}$. Finally, $A$ is valid with respect to a class of frames $\mathcal{C}(\mathcal{C} \Vdash A)$ when it is valid in all members of $\mathcal{C}$. As it is well known, cf. [5], NNML can be semantically characterized as sets of $\mathcal{L}^{\Phi}$-formulas that are valid with respect to particular classes of neighbourhood frames. In order to do so, we make use of the following notions. Given a neighbourhood frame $\mathcal{F} \equiv<W, \mathcal{N}>$, we say that:

- $\mathcal{F}$ is monotone (or supplemented) iff for all $w \in W$ and all $a, b$ in $2^{W}$, if $a \subseteq b$ and $a \in \mathcal{N}(w)$, then $b \in \mathcal{N}(w)$;

- $\mathcal{F}$ is closed under finite intersection iff for all $w \in W$ and all $a, b$ in $2^{W}$, if $a \in \mathcal{N}(w)$ and $b \in \mathcal{N}(w)$, then $a \cap b \in \mathcal{N}(w)$;

- $\mathcal{F}$ contains the unit iff for all $w \in W, W \in \mathcal{N}(w)$;

We can now summarize the characterization results for NNML, cf. [5]:

THEOREM 2.4

The logic $\mathbf{E}$ is sound and complete with respect to the class of all neighbourhood frames. EM is sound and complete with respect to the class of all monotone neighbourhood frames. EC is sound and complete with respect to the class of all neighbourhood frames closed under finite intersection. EN is sound and complete with respect to the class of all neighbourhood frames that contain the unit. Each one of EMC, EMN, and ECN is sound and complete with respect to the class of all neighbourhood frames satisfying the two relevant properties. Lastly, EMCN is sound and complete with respect to the class of all frames satisfying each one of the three properties above.

In monotone frames, the forcing condition for modal formulas can be simplified because of the following:

LEMMA 2.5

The forcing conditions below give the same class of valid formulas over monotone neighbourhood frames:

1. $w \Vdash_{1} \square A \equiv \exists a \in \mathcal{N}(w)\left(a \Vdash^{\forall} A \wedge \forall v(v \Vdash A \supset v \in a)\right)$ 


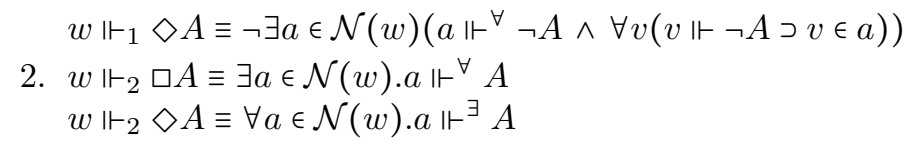

Proof. By induction on formula complexity, the only non-trivial cases being for $\square$ and $\diamond$-formulas. For the former, see [13]. For the latter, suppose that $w \Vdash_{1} \diamond A$, i.e., $\neg \exists a \epsilon$ $\mathcal{N}(w)\left(a \Vdash^{\forall} \neg A \wedge \forall v(v \Vdash \neg A \supset v \in a)\right)$. We claim that $\neg \exists a \in \mathcal{N}(w)\left(a \Vdash^{\forall} \neg A\right)$. Assume $\exists a \in \mathcal{N}(w)\left(a \Vdash^{\forall} \neg A\right)$. Since $a \subseteq \llbracket \neg A \rrbracket$, by supplementation, we have $\llbracket \neg A \rrbracket \in \mathcal{N}(w)$, therefore by choosing $\llbracket \neg A \rrbracket$ as a neighbourhood, we have $\exists a \in \mathcal{N}(w)\left(a \Vdash^{\forall} \neg A \wedge \forall v(v \Vdash\right.$ $\neg A \supset v \in a)$ ), which contradicts the assumption, therefore $\neg \exists a \in \mathcal{N}(w)\left(a \Vdash^{\forall} \neg A\right)$, i.e., $w \Vdash_{2} \diamond A$. The other direction, from $w \Vdash_{2} \diamond A$ to $w \Vdash_{1} \diamond A$, is immediate.

Moreover, when considering monotone frames we can simplify the characterization results for logics containing $C$ and $N$ as follows:

PROPOSITION 2.6

The monotone modal logic

1. EMC is sound and complete with respect the class of all monotone frames that are pre-basic: if $a, b \in \mathcal{N}(w)$, then there is $c \in \mathcal{N}(w)$ s.t.: $c \subseteq a$ and $c \subseteq b$;

2. EMN is sound and complete with respect the class of all monotone frames that are non-degenerate: for all $w \in W, \mathcal{N}(w) \neq \varnothing$.

\subsection{Labelled sequent calculi for $M M L$}

We recall from [13] the calculi for systems of non-normal logics that extend EM. Let us consider two disjoint denumerable sets of labels: a set of world labels (denoted by $w, v, u)$ and a set of neighbourhood labels (denoted by $a, b, c$ ). The labelled language is composed by:

1. Labelled formulas of shape $w: A$ (with $A$ arbitrary $\mathcal{L}^{\Phi}$-formula);

2. Additional atomic formulas of shapes (i) $w: a$, (ii) $a \in N(w),{ }^{1}$ and (iii) $a \subseteq b$;

3. Forcing formulas of shapes $a \Vdash^{\forall} A$ and $a \Vdash^{\exists} A$ (with $A$ arbitrary $\mathcal{L}^{\Phi}$-formula).

A sequent is an expression $\Gamma \Rightarrow \Delta$ where $\Gamma$ and $\Delta$ are multisets of formulas of the labelled language. The rules of the base calculus G3.M are given in Table 1, and the rules for its extensions with $C$ and $N$ are given in Table 2. In addition to the usual propositional rules of the classic labelled calculus, we have rules for the local forcing operators, for the modalities, and neighbourhood rules for the extensions that include $C$ and $N$. Such extensions are denoted by G3.M*. In Table 1, the usual rules appear for the propositional part with $P$ ranging over atomic $\mathcal{L}^{\Phi}$-formulas. Observe that $L \perp$ is listed as a zero-premiss rule rather than as initial sequent because $\perp$ is regarded as a zero-place connective. Observe also that the rules for local forcing involve quantification only implicitly in the form of variable conditions so there is no need to extend the language with quantification over possible worlds.

We observe that in the presence of rules, such as Prebasic, that introduce inclusions we also need the following rule:

$$
\frac{w \in b, w \in a, a \subseteq b, \Gamma \Rightarrow \Delta}{w \in a, a \subseteq b, \Gamma \Rightarrow \Delta} L \subseteq
$$

${ }^{1}$ Observe that we depart from the notation of [13] in using the letter $N$ instead of $I$ for the formal neighbourhoods. 
6 Proof theory for quantified monotone modal logics

TABLE 1: Rules of G3.M

\begin{tabular}{|c|c|}
\hline Initial sequents: & $w: P, \Gamma \Rightarrow \Delta, w: P$, with $P$ atomic \\
\hline \multicolumn{2}{|l|}{ Propositional rules: } \\
\hline$\frac{w: A, \Gamma \Rightarrow \Delta \quad w: B, \Gamma \Rightarrow \Delta}{w: A \vee B, \Gamma \Rightarrow \Delta} L \vee$ & $\frac{\Gamma \Rightarrow \Delta, w: A, w: B}{\Gamma \Rightarrow \Delta, w: A \vee B} R \vee$ \\
\hline$\frac{w: A, w: B, \Gamma \Rightarrow \Delta}{w: A \wedge B, \Gamma \Rightarrow \Delta} L \wedge$ & $\frac{\Gamma \Rightarrow \Delta, w: A \quad \Gamma \Rightarrow \Delta, w: B}{\Gamma \Rightarrow \Delta, w: A \wedge B} R \wedge$ \\
\hline$\frac{\Gamma \Rightarrow \Delta, w: A \quad w: B, \Gamma \Rightarrow \Delta}{w: A \supset B, \Gamma \Rightarrow \Delta} L \supset$ & $\frac{w: A, \Gamma \Rightarrow \Delta, w: B}{\Gamma \Rightarrow \Delta, w: A \supset B} R \supset$ \\
\hline$\overline{w: \perp, \Gamma \Rightarrow \Delta} L_{\perp}$ & \\
\hline \multicolumn{2}{|l|}{ Rules for modalities: } \\
\hline$\frac{b \in N(w), b \Vdash^{\forall} A, \Gamma \Rightarrow \Delta}{w: \square A, \Gamma \Rightarrow \Delta} L \square(b$ fresh $)$ & $\frac{a \in N(w), \Gamma \Rightarrow \Delta, w: \square A, a \Vdash^{\forall} A}{a \in N(w), \Gamma \Rightarrow \Delta, w: \square A}$ \\
\hline$\frac{a \Vdash^{\exists} A, a \in N(w), w: \diamond A, \Gamma \Rightarrow \Delta}{a \in N(w), w: \diamond A, \Gamma \Rightarrow \Delta} L \diamond$ & $\frac{b \in N(w), \Gamma \Rightarrow \Delta, b \Vdash^{\exists} A}{\Gamma \Rightarrow \Delta, w: \diamond A} R \diamond(b$ fresh $)$ \\
\hline Auxiliary rules: & \\
\hline$\frac{w: A, w \in a, a \Vdash^{\forall} A, \Gamma \Rightarrow \Delta}{w \in a, a \Vdash^{\forall} A, \Gamma \Rightarrow \Delta} L \Vdash^{\forall}$ & $\frac{u \in a, \Gamma \Rightarrow \Delta, u: A}{\Gamma \Rightarrow \Delta, a \Vdash^{\forall} A} R \Vdash^{\forall}(u$ fresh $)$ \\
\hline$\frac{u: A, u \in a, \Gamma \Rightarrow \Delta}{a \Vdash^{\exists} A, \Gamma \Rightarrow \Delta} L \Vdash^{\exists}(u$ fresh $)$ & $\frac{w \in a, \Gamma \Rightarrow \Delta, a \Vdash^{\exists} A, w: A}{w \in a, \Gamma \Rightarrow \Delta, a \Vdash^{\exists} A} R \Vdash^{\exists}$ \\
\hline
\end{tabular}

TABLE 2: Rules for $C$ and $N$ over G3.M

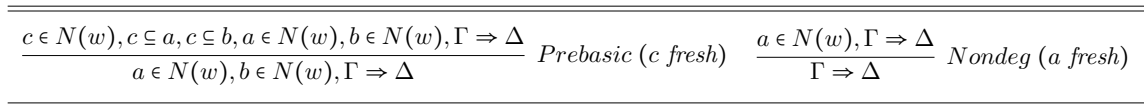

The corresponding right rule (found in [13]) instead is not needed because in the calculi that we use for monotonic extensions inclusions do not appear on the right hand side of sequents. Moreover, calculi containing rule Prebasic must contain also its contracted instances, of [15, p. 130]:

$$
\frac{a \subseteq a, a \subseteq a, a \in N(w), \Gamma \Rightarrow \Delta}{a \in N(w), \Gamma \Rightarrow \Delta} \text { Prebasic }^{c}
$$

We recall the following properties of the calculi:

Proposition 2.7 (Properties of G3.M*)

1. Sequents of the following forms are $\mathbf{G} 3 \mathbf{3} \mathbf{M}^{*}$-derivable: 
(a) $\quad a \Vdash^{\forall} A, \Gamma \Rightarrow \Delta, a \Vdash^{\forall} A$

(b) $\quad a \Vdash^{\exists} A, \Gamma \Rightarrow \Delta, a \Vdash^{\exists} A$

(c) $w: A, \Gamma \Rightarrow \Delta, w: A$

2. The following rules of substitution are height-preserving admissible in $\mathbf{G 3 .} \mathbf{M}^{*}$ :

$$
\frac{\Gamma \Rightarrow \Delta}{\Gamma[b / a] \Rightarrow \Delta[b / a]}[b / a] \quad \frac{\Gamma \Rightarrow \Delta}{\Gamma[w / v] \Rightarrow \Delta[w / v]}[w / v]
$$

3. The following rules of weakening are height-preserving admissible in $\mathbf{G 3 .} \mathbf{M}^{*}$ :

$$
\frac{\Gamma \Rightarrow \Delta}{\Gamma^{\prime}, \Gamma \Rightarrow \Delta} L W \quad \frac{\Gamma \Rightarrow \Delta}{\Gamma \Rightarrow \Delta, \Delta^{\prime}} R W
$$

4. Each rule of G3M.* is height-preserving invertible.

5. The following rules of contraction are height-preserving admissible in G3. $\mathbf{M}^{*}$ :

$$
\frac{\Gamma^{\prime}, \Gamma^{\prime}, \Gamma \Rightarrow \Delta}{\Gamma^{\prime}, \Gamma \Rightarrow \Delta} L C \quad \frac{\Gamma \Rightarrow \Delta, \Delta^{\prime}, \Delta^{\prime}}{\Gamma \Rightarrow \Delta, \Delta^{\prime}} R C
$$

6. For any formula $\phi$ of the labelled language, the following rule of Cut is admissible in $\mathbf{G 3 .} \mathbf{M}^{*}$ :

$$
\frac{\Gamma \Rightarrow \Delta, \phi \quad \phi, \Gamma^{\prime} \Rightarrow \Delta^{\prime}}{\Gamma^{\prime}, \Gamma \Rightarrow \Delta, \Delta^{\prime}} C u t
$$

7. Each calculus G3. $\mathbf{M}^{*}$ is sound and complete with respect to validity in the class of all frames for $\mathbf{M}^{*}$.

\section{Quantified monotone modal logics}

\subsection{Language}

Let $V A R$ be a denumerable set of individual variables $x, y, z, \ldots$ and let us fix a signature $\mathcal{S}$ containing, for any $n \in \mathbb{N}$, an at most denumerable set of $n$-ary relational symbols $P, Q, R \ldots$ Function symbols of any arity are omitted from $\mathcal{S}$ for the sake of simplicity. The language $\mathcal{L}^{\mathcal{S}}$ is generated by the following grammar:

$$
A::=P x_{1}, \ldots, x_{n}|x=y| \perp|A \vee A| A \wedge A|A \supset A| \square A|\diamond A| \forall x A \mid \exists x A ; \quad\left(\mathcal{L}^{\mathcal{S}}\right)
$$

where $P$ is an $n$-ary relational symbol in $\mathcal{S}$ and $x, y, x_{1}, \ldots, x_{n} \in V A R$. The same conventions and abbreviations used for $\mathcal{L}^{\Phi}$ hold, and we will use $A, B, C$ as metavariables for $\mathcal{L}^{\mathcal{S}}$-formulas (formulas, for shortness). The notions of free/bound occurrences of a variable in a formula and of open/closed formulas are as usual. We define substitution in such a way as to avoid capture of free variables while having substitutions always defined.

Definition 3.1 (Substitution)

Substitution of free variables is defined as follows:

$$
\text { 1. } z[y / x] \equiv\left\{\begin{array}{cc}
y & \text { if } z \equiv x \\
z & \text { else }
\end{array}\right.
$$


8 Proof theory for quantified monotone modal logics

2. $\left(P z_{1}, \ldots, z_{n}\right)[y / x] \equiv P z_{1}[y / x], \ldots, z_{n}[y / x] ;$

3. $\perp[y / x] \equiv \perp$;

4. $(A \circ B)[y / x] \equiv(A[y / x] \circ B[y / x]), \quad$ for $\circ \in\{\vee, \wedge, \supset\}$;

5. $(\circ A)[y / x] \equiv \circ(A[y / x]), \quad$ for $\circ \in\{\square, \diamond\} ;$

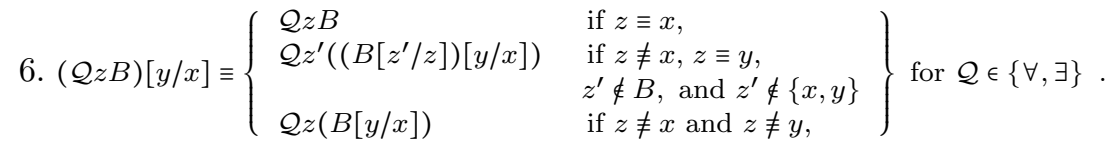

\subsection{Neighbourhood semantics for $Q M M L$}

Definition 3.2 (Frame)

A quantified monotone frame is a tuple $\mathcal{F} \equiv<W, \mathcal{N}, D, d>$ where $W$ is a non-empty set of states, $\mathcal{N}$ is a monotone neighbourhood function over $W, D$ is a non-empty set of objects, and $d: W \longrightarrow 2^{D}$ is a function mapping each state $w \in W$ to a (possibly empty) set $d(w) \subseteq D$. A constant domain frame is any quantified monotone frame such that $d(w)=D$ for all $w \in W$.

We will refer to $D$ as the outer domain of the frame, and to the $d(w)$ as the inner domains of the frame.

Definition 3.3 (Model)

Given a quantified monotone frame $\mathcal{F} \equiv\langle W, \mathcal{N}, D, d\rangle$, a model based on $\mathcal{F}$ is a tuple $<W, \mathcal{N}, D, d, \mathcal{V}>$ where $\mathcal{V}$ is a world-dependent classical first-order interpretation of the symbols in the signature $\mathcal{S}$ over the outer domain of $\mathcal{F}$. Formally, for each $n$-ary $P \in \mathcal{S}$ and each $w \in W, \mathcal{V}(P, w) \subseteq D^{n}$.

A substitution over $\mathcal{F}$ is a function $\sigma$ that maps each variable to an object of the outer domain of $\mathcal{F}$. If $\sigma$ is an assignment over $\mathcal{F}$ and $o$ is an object in $D$, we write $\sigma^{x \triangleright o}$ for the assignment that maps $x$ to $o$ and behaves like $\sigma$ for the other variables.

Definition 3.4 (Satisfaction)

The satisfaction of a $\mathcal{L}^{\mathcal{S}}$-formula $A$ at a state $w$ of a model $\mathcal{M}=\langle W, \mathcal{N}, D, d, \mathcal{V}\rangle$ (based on a monotone frame) with respect to an assignment $\sigma$, is defined by recursion on $A$ as follows (the propositional cases are as in Def. 2.3):

$$
\begin{array}{lll}
\mathcal{M}, \sigma, w \Vdash P x_{1}, \ldots, x_{n} & \text { iff } & <\sigma\left(x_{1}\right), \ldots, \sigma\left(x_{n}\right)>\in \mathcal{V}(P, w) \\
\mathcal{M}, \sigma, w \Vdash x=y & \text { iff } & \sigma(x)=\sigma(y) \\
\mathcal{M}, \sigma, w \Vdash \square B & \text { iff } & \text { there is } a \in \mathcal{N}(w) \text { s.t.: } \mathcal{M}, \sigma, v \Vdash B \text { for all } v \in a \\
\mathcal{M}, \sigma, w \Vdash \diamond B & \text { iff } & \text { for all } a \in \mathcal{N}(w) \text { there is } v \in a \text { s.t.: } \mathcal{M}, \sigma, v \Vdash B \\
\mathcal{M}, \sigma, w \Vdash \forall x B & \text { iff } & \text { for all } o \in d(w), \mathcal{M}, \sigma^{x \triangleright o}, w \Vdash B \\
\mathcal{M}, \sigma, w \Vdash \exists x B & \text { iff } & \text { there is } o \in d(w) \text { s.t.: } \mathcal{M}, \sigma^{x \triangleright o}, w \Vdash B
\end{array}
$$

Truth in a state of a model is defined as satisfaction under every assignment. The notions of global truth and of validity are defined as for the propositional case, cf. Section 2.2. 
TABLE 3: Rules for the quantifiers and for identity

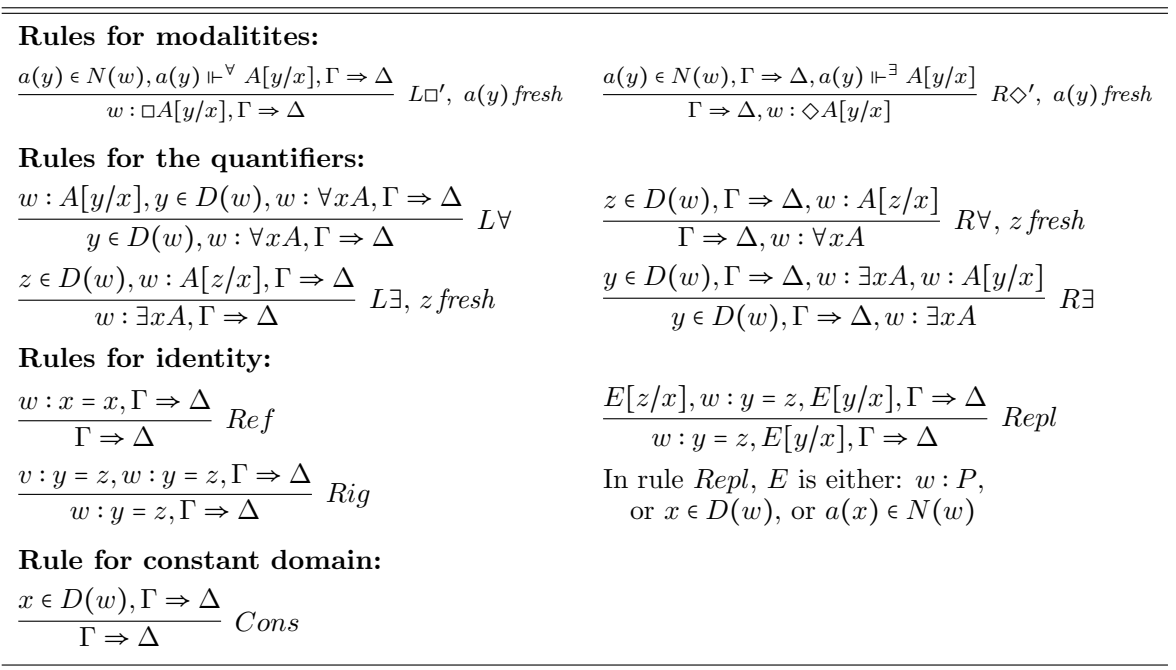

\subsection{Labelled calculi for $Q M M L$}

The calculus G3Q.M* is obtained from the calculus G3.M*, see Tables 1 (where, in initial sequents, $P$ is now an atomic formula of the first-order language $\mathcal{L}^{\mathcal{S}}$ ) and 2 , by extending the labelled language with atomic formulas of shape $x \in D(w)$ (individual variables are disjoint from both world and neighbourhood labels), and by adding the rules for the quantifiers and for identity that are given in Table 3. The rules for the quantifiers are like the rules given in [16] for varying domain relational semantics; in particular, rule Cons is added to a calculus when we want to consider its constant domain version. For identity, we have to add new atomic formulas of shape $x=y$ and (to the usual rules Ref and Repl that make = behave as a world-bound 'real identity' predicate; e.g., the rules of symmetry and transitivity for $=$ are derivable as in $[15$, Sect. 6.5]) the rule Rig that is needed because variables are rigid designators: if an assignment $\sigma$ maps two variables to the same object in one state, then it does so in all states.

Moreover, we modify the syntax of neighbourhood labels by allowing to have both simple labels of shape $a, b, c$ as well as functional labels of shape $a(x)$ where $a$ is a simple neighbourhood label and $x$ is a variable. The instances of rules $L \square^{\prime}$ and $R \diamond^{\prime}$ with functional labels are in Table 3. Notice that now rules $R \square$ and $L \diamond$ work also with respect to functional labels. ${ }^{2}$ Notice also that in rules $L \square^{\prime}$ and $R \diamond^{\prime}$ the label $a(y)$ may be fresh because of $a(\cdot)$ or because of $y$. Functional labels will not be used until we consider $B F$, thus we postpone the discussion of them to Section 5 .

\footnotetext{
${ }^{2}$ Thanks are due to an anonymous referee for noticing this fact.
} 


\section{Proof theory for quantified monotone modal logics}

\subsection{Structural properties}

In this section we shall prove the structural properties for the systems based on QMML. Our proofs build on those of [13] and the overall argument has the same structure, so we shall just recall the basic definitions of the inductive parameters to be used, suitably extend them to the quantifier language, and present in detail the new cases.

We recall that the height of a derivation is its height as a tree, i.e., the length of its longest branch minus one (so that an initial sequent has height zero), and that $\vdash_{n}$ denotes derivability with derivation height bounded by $n$ in a given system.

Definition 3.5 (Weight of a labelled formula)

The label of formulas of the form $w: A$ is $w$. The label of formulas of the form $a \Vdash^{\forall} A$, $a \Vdash^{\exists} A$ is $a$. The label of a formula $F$ will be denoted by $l(F)$. The pure part of a labelled formula $F$ is the part without the label and without the forcing relation, either local $\left(\Vdash^{\exists}, \Vdash^{\forall}\right)$ or worldwise (:) and will be denoted by $p(F)$.

The weight of a labelled formula $F$ is given by the pair $(\mathrm{w}(p(F)), \mathrm{w}(l(F)))$, where

- For all worlds labels $w$ and all neighbourhood labels $a, \mathrm{w}(w)=0$ and $\mathrm{w}(a)=1$.

- $-\mathrm{w}(P)=\mathrm{w}(\perp)=1$,

$-\mathrm{w}(A \circ B)=\mathrm{w}(A)+\mathrm{w}(B)+1$ for $\circ$ conjunction, disjunction, or implication,

$-\mathrm{w}(\square A)=\mathrm{w}(\diamond A)=\mathrm{w}(A)+1$

$-\mathrm{w}(\exists x A)=\mathrm{w}(\forall x A)=\mathrm{w}(A)+1$

For formulas of the form $x \in D(w)$ we stipulate $\mathrm{w}(x \in D(w))=(0,0)$; for formulas of the form $a \in N(w), w \in a$, we stipulate $\mathrm{w}(a \in N(w))=\mathrm{w}(w \in a)=(0,1)$; and for formulas of the form $a \subseteq b, \mathrm{w}(a \subseteq b)=(1,1)$. Weights of labelled formulas are ordered lexicographically.

From the definition of weight it is clear that the weight gets decreased if we move from a formula labelled by a neighbourhood label to the same formula labelled by a world label, or if we move (regardless the label) to a formula with a pure part of strictly smaller weight.

\section{LEMMA 3.6}

Sequents of the following forms are derivable in G3Q. $\mathbf{M}^{*}$ for arbitrary formulas $A$ in the language of quantified modal logic:

1. $a \Vdash^{\forall} A, \Gamma \Rightarrow \Delta, a \Vdash^{\forall} A$

2. $a \Vdash^{\exists} A, \Gamma \Rightarrow \Delta, a \Vdash^{\exists} A$

3. $w: A, \Gamma \Rightarrow \Delta, w: A$

Proof. Items 1, 2 are proved as in [13]. To illustrate, item 2 is proved by the following derivation, whose topmost sequent is derivable by inductive hypothesis since $\mathrm{w}(u: A)<\mathrm{w}\left(a \Vdash^{\exists} A\right)$,

$$
\frac{u: A, u \in A, \Gamma \Rightarrow \Delta, a \Vdash^{\exists} A, u: A}{\frac{u: A, u \in A, \Gamma \Rightarrow \Delta, a \Vdash^{\exists} A}{a \Vdash^{\exists} A, \Gamma \Rightarrow \Delta, a \Vdash^{\exists} A} L \Vdash^{\exists}} R \Vdash^{\exists}
$$


For 3, we supplement with the quantifier cases the proof by induction on weight given in [13] .

$A \equiv \forall x B$. We have the following inference

$$
\frac{z \in D(w), w: \forall x B, w: B[z / x], \Gamma \Rightarrow \Delta, w: B[z / x]}{\frac{z \in D(w), w: \forall x B, \Gamma \Rightarrow \Delta, w: B[z / x]}{w: \forall x B, \Gamma \Rightarrow \Delta, w: \forall x B} R \forall} L \forall
$$

where the topsequent is derivable by induction hypothesis because $\mathrm{w}(B[z / x])<\mathrm{w}(\forall x B)$.

The case with $A \equiv \exists x B$ is proved in a similar way with $L \exists$ and $R \exists$ in place of $R \forall$ and $L \forall$, respectively.

Next, we prove that the calculus enjoys the property of hp-substitution both of variables, world and (simple and functional) neighbourhood labels:

Proposition 3.7

The following hold in G3Q.M*

1. If $\vdash_{n} \Gamma \Rightarrow \Delta$, then $\vdash_{n} \Gamma[b \cdot / a \cdot] \Rightarrow \Delta[b \cdot / a \cdot] \quad$ where $b \cdot(a \cdot$, respectively) might be either $b$ or $b(y)$ ( $a$ or $a(y)$ respectively), for some variable $y$;

2. If $\vdash_{n} \Gamma \Rightarrow \Delta$, then $\vdash_{n} \Gamma[y / x] \Rightarrow \Delta[y / x]$;

3. If $\vdash_{n} \Gamma \Rightarrow \Delta$, then $\vdash_{n} \Gamma[v / w] \Rightarrow \Delta[v / w]$.

Proof. All three statements are proved by induction on the height of the derivation. Notice that, since the substitution of variables may have impact on functional neighbourhood labels, it is essential to prove the admissibility of substitution of functional neighbourhood labels before that of variables in order to avoid clashes with variable condition.

If the height is $0, \Gamma \Rightarrow \Delta$ is an initial sequent or a conclusion of $L \perp$. The same then holds for $\Gamma[y / x] \Rightarrow \Delta[y / x]$, for $\Gamma[v / w] \Rightarrow \Delta[v / w]$, and for $\Gamma[b \cdot / a \cdot] \Rightarrow \Delta[b \cdot / a \cdot]$.

If the derivation has height $n>0$, we consider the last rule applied. If $\Gamma \Rightarrow \Delta$ has been derived by a rule without variable conditions, we apply the induction hypothesis and then the rule. Rules with variable conditions require that we avoid a clash of the substituted variable with the fresh variable in the premiss. This is the case for the logical rules $L \square^{\left({ }^{\prime}\right)}, \mathrm{R} \diamond^{\left(^{\prime}\right)} R \Vdash^{\forall}, L \Vdash^{\exists}, L \exists, R \forall$ and for the neighbourhood rule Nondeg. So, if $\Gamma \Rightarrow \Delta$ has been derived by one of these rules, we apply the inductive hypothesis twice to the premiss, first to replace the fresh variable with another fresh variable, different, if necessary, from the one we want to substitute, then to make the substitution, and finally we apply the rule. In cases where the last step is by $L \square^{\prime}\left(R \diamond^{\prime}\right)$ with a variable condition on $a(x)$ and the variable substitution $[y / x]$ would clash with its variable condition, we start by applying the hp-admissible substitution $[b(x) / a(x)]$ for some $b(x)$ such that neither it nor $b(y)$ occur in the sequent, then we apply the inductive hypothesis and the rule.

The rules of weakening for the language of a labelled system with internalized neighbourhood semantics such as G3Q.M ${ }^{*}$ have the following form, where $\phi$ is either a "relational" atom of the form $a \in N(w), w \in a$, or $x \in D(w),{ }^{3}$ or a labelled formula of the form $w: A, a \Vdash^{\forall} A, a \Vdash^{\exists} A$ :

$$
\frac{\Gamma \Rightarrow \Delta}{\phi, \Gamma \Rightarrow \Delta} L-W k n \quad \frac{\Gamma \Rightarrow \Delta}{\Gamma \Rightarrow \Delta, \phi} R-W k n
$$

${ }^{3}$ Indeed, such formulas are not needed for right weakenening because they are never active on the right. 


\section{Proof theory for quantified monotone modal logics}

Proposition 3.8

The structural rules of left and right weakening are hp-admissible in G3Q.M* .

Proof. Straightforward induction, with a similar proviso as in the above proof for rules with variable conditions.

Next, we prove $h p$-invertibility of the rules of G3Q.M ${ }^{*}$, i.e., for every rule of the form $\frac{\Gamma^{\prime} \Rightarrow \Delta^{\prime}}{\Gamma \Rightarrow \Delta}$, if $\vdash_{n} \Gamma \Rightarrow \Delta$ then $\vdash_{n} \Gamma^{\prime} \Rightarrow \Delta^{\prime}$, and for every rule of the form $\frac{\Gamma^{\prime} \Rightarrow \Delta^{\prime} \quad \Gamma^{\prime \prime} \Rightarrow \Delta^{\prime \prime}}{\Gamma \Rightarrow \Delta}$, if $\vdash_{n} \Gamma \Rightarrow \Delta$ then $\vdash_{n} \Gamma^{\prime} \Rightarrow \Delta^{\prime}$ and $\vdash_{n} \Gamma^{\prime \prime} \Rightarrow \Delta^{\prime \prime}$ :

LEMMa 3.9

The following hold in G3Q.M* :

1. If $\vdash_{n} \Gamma \Rightarrow \Delta, a \Vdash^{\forall} A$ then $\vdash_{n} w \in a, \Gamma \Rightarrow \Delta, w: A$.

2. If $\vdash_{n} w \in a, a \Vdash^{\forall} A, \Gamma \Rightarrow \Delta$ then $\vdash_{n} w \in a, w: A, a \Vdash^{\forall} A, \Gamma \Rightarrow \Delta$.

3. If $\vdash_{n} w \in a, \Gamma \Rightarrow \Delta, a \Vdash^{\exists} A$ then $\vdash_{n} w \in a, \Gamma \Rightarrow \Delta, w: A, a \Vdash^{\exists} A$.

4. If $\vdash_{n} a \Vdash^{\exists} A, \Gamma \Rightarrow \Delta$ then $\vdash_{n} w \in a, w: A, \Gamma \Rightarrow \Delta$.

5. If $\vdash_{n} w: \square A, \Gamma \Rightarrow \Delta$ then $\vdash_{n} a \in N(w), a \Vdash^{\forall} A, \Gamma \Rightarrow \Delta$.

6. If $\vdash_{n} a \in N(w), \Gamma \Rightarrow \Delta, w: \square A$ then $\vdash_{n} a \in N(w), \Gamma \Rightarrow \Delta, w: \square A, a \Vdash^{\forall} A$.

7. If $\vdash_{n} a \in N(w), w: \diamond A, \Gamma \Rightarrow \Delta$ then $\vdash_{n} a \Vdash^{\exists} A, a \in N(w), w: \diamond A, \Gamma \Rightarrow \Delta$.

8. If $\vdash_{n} \Gamma \Rightarrow \Delta, w: \diamond A$ then $\vdash_{n} a \in N(w), \Gamma \Rightarrow \Delta, a \Vdash^{\exists} A$.

9. If $\vdash_{n} w \in a, a \subseteq b, \Gamma \Rightarrow \Delta$ then $\vdash_{n} w \in a, a \subseteq b, w \in b, \Gamma \Rightarrow \Delta$.

10. If $\vdash_{n} y \in D(w), w: \forall x A, \Gamma \Rightarrow \Delta$ then $\vdash_{n} w: A[y / x], y \in D(w), w: \forall x A, \Gamma \Rightarrow \Delta$.

11. If $\vdash_{n} \Gamma \Rightarrow \Delta, w: \forall x A$ then $\vdash_{n} z \in D(w), \Gamma \Rightarrow \Delta, w: A[z / x]$.

12. If $\vdash_{n} w: \exists x A, \Gamma \Rightarrow \Delta$ then $\vdash_{n} z \in D(w), w: A[z / x], \Gamma \Rightarrow \Delta$.

13. If $\vdash_{n} y \in D(w), \Gamma \Rightarrow \Delta, w: \exists x A$ then $\vdash_{n} y \in D(w), \Gamma \Rightarrow \Delta, w: \exists x A, w: A[y / x]$.

14. If $\vdash_{n} \Gamma \Rightarrow \Delta$ then $\vdash_{n} w: x=x, \Gamma \Rightarrow \Delta$.

15. If $\vdash_{n} w: y=z, E[y / x], \Gamma \Rightarrow \Delta$ then $\vdash_{n} E[z / x], w: y=z, E[y / x], \Gamma \Rightarrow \Delta$.

16. If $\vdash_{n} w: y=z, \Gamma \Rightarrow \Delta$ then $\vdash_{n} v: y=z, w: y=z, \Gamma \Rightarrow \Delta$.

17. For systems containing rule Cons, if $\vdash_{n} \Gamma \Rightarrow \Delta$ then $\vdash_{n} x \in D(w), \Gamma \Rightarrow \Delta$.

18. For systems containing rule Prebasic, if $\vdash_{n} a \in N(w), b \in N(w), \Gamma \Rightarrow \Delta$ then $\vdash_{n} c \in N(w), c \subseteq a, c \subseteq b, a \in N(w), b \in N(w), \Gamma \Rightarrow \Delta$.

19. For systems containing rule Nondeg, if $\vdash_{n} \Gamma \Rightarrow \Delta$ then $\vdash_{n} a \in N(w), \Gamma \Rightarrow \Delta$.

Proof. Similar to the proof in [13], observing first that all the cases that are instances of hp-admissibility of weakening (i.e., all cases but 1, 4, 5, 8, 11, and 12) follow from Proposition 3.8 above. For the rest, the proof is by induction on $n$, considering the last rule applied in a derivation. We prove only case 8 , all others being similar.

For the base case, assume that $\vdash^{0} \Gamma \Rightarrow \Delta, w: \diamond A$, then, for some label $v$, either some atomic $v: P$ occurs in both $\Gamma$ and $\Delta$ or $v: \perp$ occurs in $\Gamma$. In both cases we have that $\vdash^{0} a \in N(w), \Gamma \Rightarrow \Delta, a \Vdash^{\exists} A$.

For the inductive step, we assume that the lemma holds up to derivation-height $n$ and we assume that $\vdash^{n+1} \Gamma \Rightarrow \Delta, w: \diamond A$. If the displayed instance of $w: \diamond A$ is principal in the last step, we have (possibly using Proposition 3.7) a derivation of height $n$ of $a \in N(w), \Gamma \Rightarrow \Delta, a \Vdash^{\exists} A$ and we are done. Else, the last step is by some rule $R$ with either one or two premisses and, possibly, with a variable condition. We consider only 
the critical case of a rule $R$ with one premiss $\Gamma^{\prime} \Rightarrow \Delta^{\prime}, w: \diamond A$ and a variable condition on $a$. The premiss has a derivation of height $n$. We apply to it an height-preserving admissible substitution, Proposition 3.7, to obtain $\vdash^{n} \Gamma^{\prime}[b / a] \Rightarrow \Delta^{\prime}[b / a], w: \diamond A$, for some fresh neighbourhood label $b$. By induction we know the lemma holds for derivations of height $n$, and therefore we have $\vdash^{n} a \in N(w), \Gamma^{\prime}[b / a] \Rightarrow \Delta^{\prime}[b / a], a \Vdash^{\exists} A$. By an instance of rule $R$ we conclude $\vdash^{n+1} a \in N(w), \Gamma \Rightarrow \Delta, a \Vdash^{\exists} A$.

Since all the propositional rules of G3Q.M $\mathbf{M}^{*}$ are easily shown to be hp-invertible, we have:

Corollary 3.10

All the rules of G3Q. $\mathbf{M}^{*}$ are hp-invertible.

The rules of contraction for the language of a labelled system with internalized neighbourhood semantics such as G3Q. $\mathbf{M}^{*}$ have the following form, where $\phi$ is either a "relational" atom of the form $a \in N(w), w \in a$, or $x \in D(w)$, or a labelled formula of the form $w: A, a \Vdash^{\forall} A, a \Vdash^{\exists} A$ :

$$
\frac{\phi, \phi, \Gamma \Rightarrow \Delta}{\phi, \Gamma \Rightarrow \Delta} L_{-C t r} \quad \frac{\Gamma \Rightarrow \Delta, \phi, \phi}{\Gamma \Rightarrow \Delta, \phi} R-C t r
$$

THEOREM 3.11

The rules of left and right contraction are hp-admissible in G3Q.M* .

Proof. Similar to the proof in [13], by simultaneous induction on the height of derivation for left and right contraction.

In the base case the premiss is either an instance of an initial sequent or a conclusion of $L \perp$, and so is the conclusion. In the inductive step we have to consider the last rule (instance) $R$ used in the derivation of the premiss. If the contraction formula $\phi$ is not principal in $R$, we can simply contract the instances of $\phi$ that are in the premiss(es) (since the premiss(es) has(have) lower height), and then we conclude with an instance of rule $R$. Else, (at least) one instance of the contraction formula is principal in $R$. If $R$ is an instance of a rule with repetition of the principal formula(s) we proceed as in the case above. ${ }^{4}$ Finally, if $\phi$ is principal in $R$ and $R$ is an instance of a rule without repetition of the principal formula(s), we begin by applying invertibility to the premiss(es) of $R$, then we apply the inductive hypothesis, and we conclude by an instance of $R$.

Cut is a rule of the form

$$
\frac{\Gamma \Rightarrow \Delta, \phi \quad \phi, \Gamma^{\prime} \Rightarrow \Delta^{\prime}}{\Gamma, \Gamma^{\prime} \Rightarrow \Delta, \Delta^{\prime}} C u t
$$

where $\phi$ is any formula of the language of the labelled calculus $\mathbf{G 3 Q} \cdot \mathbf{M}^{\star}$. We have:

THEOREM 3.12

Cut is admissible in G3Q.M* .

\footnotetext{
${ }^{4}$ Note that, if $R$ is an instance of Prebasic, it might be that we have to conclude with an instance of its contracted

version $\frac{a \subseteq a, a \subseteq a, a \in N(w), \Gamma \Rightarrow \Delta}{a \in N(w), \Gamma \Rightarrow \Delta}$ Prebasic $^{c}$. No other rule considered in the paper needs a contracted version.
} 


\section{Proof theory for quantified monotone modal logics}

Proof. By double induction, with primary induction on the weight of the cut formula and subinduction on the cut height, i.e., the sum of the heights of derivations of the premisses of cut. The cases in which the premisses of cut are either initial sequents or obtained through the rules for $\wedge, \vee$, or $\supset$ follow the treatment of Theorem 3.2.3 of [15] since in the calculus initial sequents are limited to atomic formulas of the form $w: P$, with the exception that there are rules for equality with principal atomic formulas. However, such cases do not cause any extra trouble since a cut with an initial sequent produces a sequent with the same conclusion modulo a weakening in the context, and thus can be replaced by admissible weakening steps.

For the cases in which the cut formula is a side formula in at least one rule used to derive the premisses of cut, the cut reduction is dealt with in the usual way by permutation of cut, with possibly an application of hp-substitution to avoid a clash with the fresh variable in rules with variable condition. In all such cases the cut height is reduced. We just give an example of such a reduction, all the other cases being similar:

$$
\frac{\Gamma \Rightarrow \Delta, \phi \frac{a \in N(w), a \Vdash^{\forall} A, \phi, \Gamma^{\prime} \Rightarrow \Delta^{\prime}}{w: \square A, \phi, \Gamma^{\prime} \Rightarrow \Delta^{\prime}}}{w: \square A, \Gamma, \Gamma^{\prime} \Rightarrow \Delta, \Delta^{\prime}} L
$$

the neighbourhood label $a$ occurring in the premiss of $L \square$ is fresh, but nothing prevents it from appearing in the left premiss of cut; therefore, prior to the permutation of cut, we need to replace it with a neighbourhood label which is fresh not just with respect to the conclusion of $L \square$ but also with respect to the left premiss of cut. Let the new fresh variable be $c$. The transformed derivation, with cut reduced to a cut of smaller height, is as follows:

$$
\frac{\Gamma \Rightarrow \Delta, \phi \quad c \in N(w), c \Vdash^{\forall} A, \phi, \Gamma^{\prime} \Rightarrow \Delta^{\prime}}{\frac{c \in N(w), c \Vdash^{\forall} A, \Gamma, \Gamma^{\prime} \Rightarrow \Delta, \Delta^{\prime}}{w: \square A, \Gamma, \Gamma^{\prime} \Rightarrow \Delta, \Delta^{\prime}} L \square} C u t
$$

The cases with cut formula principal in both premisses of cut and either propositional or of the form $a \Vdash^{\forall} A, a \Vdash^{\exists} A, w: \square A$, and $w: \diamond A$ are dealt as in [13] (with the monotonic version of the rules for $\square$ and $\diamond$ ). To illustrate, if $\phi$ is $w: \diamond A$, we have:

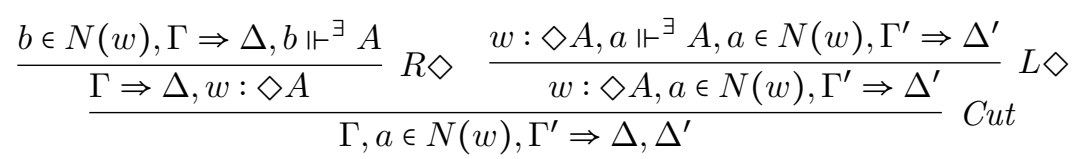

which can be transformed into the following derivation having two smaller cuts, the upper one with lower height, and the lower one with a cut formula of reduced weight:

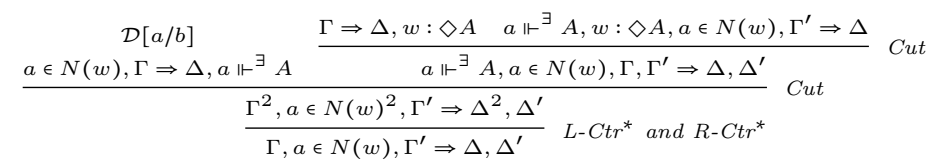

If the cut formula is $w: \forall x A$, principal in both premisses of cut,

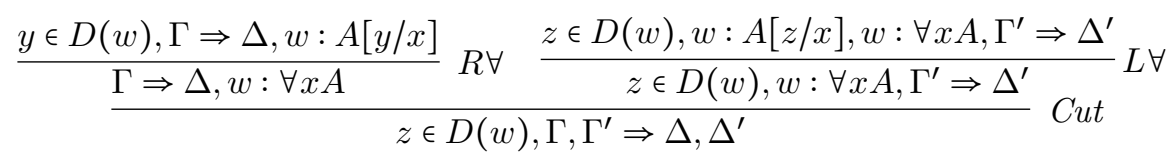


This is transformed into a derivation with two smaller cuts, the upper one with the original cut formula but smaller derivation height, and the lower one with a cut formula of reduced weight:

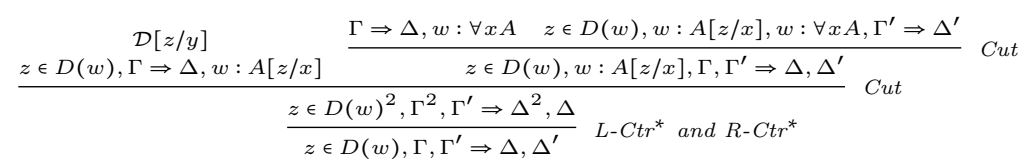

For cut formula of the form $\exists x A$ principal in both premisses of cut, the reduction is similar to the above.

\section{Soundness and completeness}

Next, we give a proof of soundness and completeness of our calculi with respect to quantified monotone frames. Specifically, we show that all the rules are sound, and we show that proof search in each calculus either produces a proof, or provides us with a saturated branch which is used to define a countermodel in the appropriate class of quantified monotone frames.

The truth of a formula in the labelled system with respect to a model $\mathcal{M}=$ $<W, \mathcal{N}, D, d, \mathcal{V}>$ depends on assignments of syntactic components in the models, so not just variables but also labels for possible worlds and neighbourhoods. We thus need to extend the notion of $S N$-realisation (introduced in [14]) with assignments for first-order variables in a quantified neighbourhood model:

DEFinition 4.1 ( $S N$-realization)

Given a set $S$ of world labels $w$ and a set $N L$ of neighbourhood labels $a$, and a neighbourhood model $\mathcal{M}=(W, \mathcal{N}, D, d, \mathcal{V})$, an $S N$-realisation $(\rho, \nu)$ is a pair of functions mapping each $w \in S$ into $\rho(w) \in W$ and mapping each $a \in N L$ into $\nu(a) \in \mathcal{N}(w)$ for some $w \in W$. We introduce the notion ' $\mathcal{M}$ satisfies a formula $F$ under an $S N$ realisation $(\rho, \nu)$ and an assignment of variables $\sigma$ ' and denote it by $\mathcal{M} \vDash_{\rho, \nu, \sigma} F$, where we assume that the labels in $F$ occur in $S, N L$.

$$
\begin{aligned}
& \text { - } \mathcal{M} \vDash_{\rho, \nu, \sigma} w \in a \quad \text { iff } \quad \rho(w) \in \nu(a) \\
& \text { - } \mathcal{M} \vDash_{\rho, \nu, \sigma} a \in N(w) \quad \text { iff } \quad \nu(a) \in \mathcal{N}(\rho(w)) \\
& \text { - } \mathcal{M} \vDash_{\rho, \nu, \sigma} x \in D(w) \quad \text { iff } \sigma(x) \in d(\rho(w)) \\
& \text { - } \mathcal{M} \vDash_{\rho, \nu, \sigma} a \subseteq b \quad \text { iff } \nu(a) \subseteq \nu(b) \\
& \text { - } \mathcal{M} \vDash_{\rho, \nu, \sigma} w: A \quad \text { iff } \mathcal{M}, \sigma, \rho(w) \Vdash A \quad \text { (see Definition 3.4) } \\
& \text { - } \mathcal{M} \vDash_{\rho, \nu, \sigma} a \Vdash^{\exists} A \quad \text { iff } \quad \exists v \in W(v \in \nu(a) \wedge \mathcal{M}, \sigma, v \Vdash A) \\
& \text { - } \mathcal{M} \vDash_{\rho, \nu, \sigma} a \Vdash^{\forall} A \quad \text { iff } \quad \forall v \in W(v \in \nu(a) \supset \mathcal{M}, \sigma, v \Vdash A)
\end{aligned}
$$

Given a sequent $\Gamma \Rightarrow \Delta$, let $S, N L$ be the sets of world and neighbourhood labels occurring in $\Gamma \cup \Delta$, and let $(\rho, \nu)$ be an $S N$-realisation and $\sigma$ an assignment of variables, we define: $\mathcal{M} \vDash_{\rho, \nu, \sigma} \Gamma \Rightarrow \Delta$ if whenever $\mathcal{M} \vDash_{\rho, \nu, \sigma} F$ for all formulas $F \in \Gamma$ then $\mathcal{M} \vDash_{\rho, \nu, \sigma} G$ for some formula $G \in \Delta$. We further define $\mathcal{M}$-validity by:

$\mathcal{M} \vDash \Gamma \Rightarrow \Delta$ iff $\mathcal{M} \vDash_{\rho, \nu, \sigma} \Gamma \Rightarrow \Delta$ for every $S N$-realisation $(\rho, \nu)$ and every assignment $\sigma$.

We say that a sequent $\Gamma \Rightarrow \Delta$ is valid in a quantified monotone neighbourhood frame $\mathcal{F}$ if $\mathcal{M} \vDash \Gamma \Rightarrow \Delta$ for every quantified monotone neighbourhood model $\mathcal{M}$ based on 
$\mathcal{F}$. Finally, we say that a sequent $\Gamma \Rightarrow \Delta$ is $\mathbf{Q}$.L-valid if it is valid in all quantified monotone neighbourhood frames that are frames for the logic $\mathbf{L}$.

\section{Soundness}

Below, we shall use the notation $\mathcal{M} \vDash_{\rho, \nu, \sigma} \Gamma$ for $\mathcal{M} \vDash_{\rho, \nu, \sigma} F$ for all $F \in \Gamma$, where $\vDash_{\rho, \nu, \sigma}$ is the notion of truth introduced in Definition 4.1.

THEOREM 4.2

If $\Gamma \Rightarrow \Delta$ is derivable in G3Q.M, then it is valid in the class of quantified monotone neighbourhood frames. In the extensions with rule Prebasic and Nondeg, validity holds with respect to pre-basic and non-degenerate frames, respectively. In the extensions with rule Cons, validity holds with respect to constant domain frames.

Proof. By induction on the height $n$ of the derivation of $\Gamma \Rightarrow \Delta$ in G3Q.M*

For $n=0$, observe that initial sequents have the same labelled formula in the antecedent and in the succedent so the claim is obvious. Similarly if the antecedent contains $w: \perp$ because we assume that for no $w \in W$ and no assignment $\sigma$ we have that $\mathcal{M}, \sigma, w \Vdash \perp$.

For the inductive step, consider the last rule in the derivation of $\Gamma \Rightarrow \Delta$. If it is a propositional rule, the claim is immediate by the definition of the forcing clauses for the propositional connectives.

If the last rule is $R \Vdash^{\forall}$, assume by induction hypothesis that $u \in a, \Gamma \Rightarrow \Delta, u: A$ is valid. Let $(\rho, \nu)$ be an arbitrary $S N$-realisation and $\sigma$ an arbitrary assignment for the conclusion and assume that $\mathcal{M} \vDash_{\rho, \nu, \sigma} \Gamma$. Since $u$ is fresh, $\rho$ can be extended to $\rho^{\prime}$, an $S$-realization for the premiss with $\rho^{\prime}(u) \in \nu(a)$. Then (using the assumption that $u \notin \Gamma$ ) we have $\mathcal{M} \vDash_{\rho^{\prime}, \nu, \sigma} u \in a, \Gamma$. By the hypothesis $\mathcal{M} \vDash u \in a, \Gamma \Rightarrow \Delta, u: A$, we have that either (1) $\mathcal{M} \vDash_{\rho^{\prime}, \nu, \sigma} G$ for some $G$ in $\Delta$ or $(2) \mathcal{M} \vDash_{\rho^{\prime}, \nu, \sigma} u: A$. In the former case we are done, so let us assume that $\mathcal{M} \vDash_{\rho^{\prime}, \nu, \sigma} G$ for no $G$ in $\Delta$. Since $u \notin \Delta$, this will be the case uniformly, independently of the choice of $\rho^{\prime}(u)$, so we'll have $\mathcal{M} \vDash_{\rho^{\prime}, \nu, \sigma} u: A$ for all $\rho^{\prime}(u) \in \nu(a)$, and therefore $\mathcal{M} \vDash_{\rho, \nu, \sigma} a \Vdash^{\forall} A$.

If the last rule is $L \Vdash^{\forall}$, the claim holds since if $\mathcal{M} \vDash_{\rho, \nu, \sigma} w \in a$ and $\mathcal{M} \vDash_{\rho, \nu, \sigma} a \Vdash^{\forall} A$, then $\mathcal{M} \vDash_{\rho, \nu, \sigma} w: A$ by simply unfolding the definitions.

If the last rule is $R \Perp^{\exists}$, consider an arbitrary $S N$-realisation $(\rho, \nu)$ and an arbitrary assignment $\sigma$, and assume that (1) $\mathcal{M} \vDash_{\rho, \nu, \sigma} w \in a, \Gamma$. Then, by induction hypothesis, either (2) $\mathcal{M} \vDash_{\rho, \nu, \sigma} G$ for some $G \in \Delta$, or (3) $\mathcal{M} \vDash_{\rho, \nu, \sigma} w: A$, or (4) $\mathcal{M} \vDash_{\rho, \nu, \sigma} a \Vdash^{\exists} A$. If (2) or (4) hold, then the claim follows. If (3) holds, we have $\rho(w) \Vdash A$. Observe that (1) gives in particular $\rho(w) \in \nu(a)$, so there is $u \in \nu(a)$ such that $\mathcal{M}, \sigma, u \Vdash A$, i.e. $\nu(a) \Vdash^{\exists} A$. It follows that the conclusion of the rule is $\mathcal{M}$-valid for the $S N$-realization $(\rho, \nu)$ and the assignment $\sigma$.

If the last rule is $L \Vdash^{\exists}$, assume that $\mathcal{M} \vDash_{\rho, \nu, \sigma} a \Vdash^{\exists} A, \Gamma$ for an arbitrary $S N$ realisation for the conclusion $(\rho, \nu)$ and assignment $\sigma$. Then there is $w \in \nu(a)$ such that $\mathcal{M}, \sigma, w \Vdash A$. Since $u$ is fresh, we can extend $\rho$ to an $S$-realization for the premiss by choosing $\rho^{\prime}(u)=w$. Then we have $\mathcal{M} \vDash_{\rho^{\prime}, \nu, \sigma} u \in a, u: A$ by definition, and $\mathcal{M} \vDash_{\rho^{\prime}, \nu, \sigma} \Gamma$ because $u \notin \Gamma$. By induction hypothesis, the premiss of the rule is $\mathcal{M}$-valid, and therefore there is $G$ in $\Delta$ such that $\mathcal{M} \vDash_{\rho^{\prime}, \nu, \sigma} G$. Since $u \notin \Delta$, this is the same as $\mathcal{M} \vDash_{\rho, \nu, \sigma} G$.

If the last rule is $L \square$, assume the premiss valid and let $(\rho, \nu)$ be an arbitrary $S N$ realisation and $\sigma$ an arbitrary assignment with $\mathcal{M} \vDash_{\rho, \nu, \sigma} w: \square A, \Gamma$. This means that 
there is $\alpha$ in $N(\rho(w))$ with $\alpha \subseteq \llbracket A \rrbracket_{\sigma}$. Since $a$ is fresh, we can extend $\nu$ to $\nu^{\prime}$ by having $\nu^{\prime}(a)=\alpha$. We have $\mathcal{M} \vDash_{\rho, \nu^{\prime}, \sigma} a \in N(w), a \Vdash^{\forall} A$ by the definitions and also $\mathcal{M} \vDash_{\rho, \nu^{\prime}, \sigma} \Gamma$ because $a \notin \Gamma$ and by hypothesis $\mathcal{M} \vDash_{\rho, \nu, \sigma} \Gamma$. Again by hypothesis, there is $B$ in $\Delta$ with $\mathcal{M} \vDash_{\rho, \nu^{\prime}, \sigma} B$ and thus by freshness of $a$ we have $\mathcal{M} \vDash_{\rho, \nu, \sigma} B$.

If the last rule is $R \square$, assume the premiss valid and assume for an arbitrary $S N$ realisation $(\rho, \nu)$ and assignment $\sigma$ that $\mathcal{M} \vDash_{\rho, \nu, \sigma} a \in N(w), \Gamma$. From the validity of the premiss we have that one of the following alternatives holds: $1: \mathcal{M} \vDash_{\rho, \nu, \sigma} B$ for some $B$ in $\Delta$. $2 . \mathcal{M} \vDash_{\rho, \nu, \sigma} w: \square A$. 3. $\mathcal{M} \vDash_{\rho, \nu, \sigma} a \Vdash^{\forall} A$. Observe that the latter gives, together with $\mathcal{M} \vDash_{\rho, \nu, \sigma} a \in N(w)$ that $\mathcal{M} \vDash_{\rho, \nu, \sigma} w: \square A$ so in each of the three cases we have proved the claim.

If the last rule is $L \diamond$, assume the premiss valid and assume for an arbitrary $S N$ realisation $(\rho, \nu)$ and assignment $\sigma$ that $\mathcal{M} \vDash_{\rho, \nu, \sigma} a \in N(w), w: \diamond A, \Gamma$. Then $\mathcal{M} \vDash_{\rho, \nu, \sigma}$ $a \Vdash^{\exists} A$ and by the hypothesis we conclude that $\mathcal{M} \vDash_{\rho, \nu, \sigma} B$ for some $B \in \Delta$.

If the last rule is $R \diamond$ assume $\mathcal{M} \vDash b \in N(w), \Gamma \Rightarrow \Delta, b \Vdash^{\exists} A$. Let $(\rho, \nu)$ be an arbitrary $S N$-realization and $\sigma$ be an arbitrary assignment such that $\mathcal{M} \vDash_{\rho, \nu, \sigma} \Gamma$. We extend $\nu$ to an $N$-realization $\nu^{\prime}$ such that $\nu^{\prime}(b) \in \mathcal{N}(\rho(w))$. Since $B$ is not in $\Gamma$, we have $\mathcal{M} \vDash_{\rho, \nu^{\prime}, \sigma} b \in N(w), \Gamma$ and by the hypothesis either $\mathcal{M} \vDash_{\rho, \nu^{\prime}, \sigma} B$ with $B \in \Delta$ or $\mathcal{M} \vDash_{\rho, \nu^{\prime}, \sigma} b \Vdash^{\exists} A$. If the former holds we are done (thanks to the freshness of $b$ ). Else, independently of the choiche of $\nu^{\prime}(b)$, there is $\rho(u) \in \nu^{\prime}(b)$ such that $\mathcal{M} \vDash_{\rho, \nu^{\prime}, \sigma} u: B$, and therefore $\mathcal{M} \vDash_{\rho, \nu, \sigma} w: \diamond A$.

The case of rule $L \square^{\prime}\left(R \diamond^{\prime}\right)$ is similar to $L \square(R \diamond)$ and can be omitted.

If the last rule is $L \forall$, assume the premiss valid. Let $(\rho, \nu)$ be an arbitrary $S N$ realisation and $\sigma$ an arbitrary assignment such that $\mathcal{M} \vDash_{\rho, \nu, \sigma} y \in D(w), w: \forall x A, \Gamma$. Then in particular $\mathcal{M} \vDash_{\rho, \nu, \sigma} w: \forall x A$, i.e., for all $o \in d(\sigma(w)), \mathcal{M} \vDash_{\rho, \nu, \sigma^{x \triangleright o}} A$. Since $\mathcal{M} \vDash_{\rho, \nu, \sigma} y \in D(w)$, we have $\mathcal{M} \vDash_{\rho, \nu, \sigma} w: A[y / x]$. Thus all the formulas in the antecedent of the premiss are validated in $(\rho, \nu, \sigma)$ and by inductive hypothesis there is $F$ in $\Delta$ such that $\mathcal{M} \vDash_{\rho, \nu, \sigma} F$, so the conclusion is valid.

If the last rule is $R \forall$, assume the premiss $z \in D(w), \Gamma \Rightarrow \Delta, w: A[z / x]$ valid. Let $(\rho, \nu)$ be an arbitrary $S N$-realisation and $\sigma$ an arbitrary assignment that validates all the formulas in $\Gamma$. We claim that one of the formulas in $\Delta$ or $w: \forall x A$ is valid under $(\rho, \nu, \sigma)$. Let $z \in d(\rho(w))$ and $\sigma^{\prime} \equiv \sigma^{x \triangleright z}$. We have $\mathcal{M} \vDash_{\rho, \nu, \sigma^{\prime}} z \in D(w)$, $\Gamma$, so by inductive hypothesis $\mathcal{M} \vDash_{\rho, \nu, \sigma^{\prime}} F$ for some $F$ in $\Delta$ or $\mathcal{M} \vDash_{\rho, \nu, \sigma^{\prime}} w: A[z / x]$. In the former case, $\mathcal{M} \vDash_{\rho, \nu, \sigma} F$ (because $z$ is fresh), in the latter, $\mathcal{M} \vDash_{\rho, \nu, \sigma} \forall x A$.

The cases of $L \exists$ and $R \exists$ are similar, respectively, to $R \forall$ and $L \forall$.

If the last rule is Ref, assume by induction hypothesis that $\mathcal{M} \vDash w: x=x, \Gamma \Rightarrow$ $\Delta$. Let $(\rho, \nu)$ be an arbitrary $S N$-realisation and $\sigma$ an arbitrary assignment for the conclusion and assume that $\mathcal{M} \vDash_{\rho, \nu, \sigma} \Gamma$. Since $\sigma(x)=\sigma(x)$, we have $\mathcal{M} \vDash w: x=x, \Gamma$. The assumption gives $\mathcal{M} \vDash F$ for some $F \in \Delta$, so the conclusion of the rule is valid.

If the last rule is $R e p l$, assume $\mathcal{M} \vDash w: y=z, E[y / x], \Gamma$. Since we have $\sigma(y)=$ $\sigma(z)$ and $\mathcal{M} \vDash E[y / x]$, also $\mathcal{M} \vDash E[z / x]$ holds. Inductive hypothesis gives the conclusion. Validity of Rig is straightforward. It is also straightforward to show that rules Prebasic, Nondeg and Cons preserve validity over quantified monotone models in the corresponding classes. 


\section{Proof theory for quantified monotone modal logics}

\section{Completeness}

Next, we move to completeness. First, we give the definition of saturated branch. Intuitively, a branch is saturated when, in root-first proof search, all the applicable rules have been applied.

DEFINITION 4.3 (Saturation)

A branch in a proof search from the endsequent up to a sequent $\Gamma \Rightarrow \Delta$ is saturated with respect to a rule $R$ if condition $(R)$ below holds, where we indicate with $\downarrow \Gamma(\downarrow \Delta)$ the union of the antecedents (succedents) in the branch from the end-sequent up to $\Gamma \Rightarrow \Delta:$

(Init) There is no $w: P$ in $\Gamma \cap \Delta$.

$(\mathrm{L} \perp)$ There is no $w: \perp$ in $\Gamma$.

$(\mathrm{L} \wedge)$ If $w: A \wedge B$ is in $\downarrow \Gamma$, then $w: A$ and $w: B$ are in $\downarrow \Gamma$.

$(\mathrm{R} \wedge)$ If $w: A \wedge B$ is in $\downarrow \Delta$, then either $w: A$ or $w: B$ is in $\downarrow \Delta$.

(Lv) If $w: A \vee B$ is in $\downarrow \Gamma$, then either $w: A$ or $w: B$ is in $\downarrow \Gamma$.

(Rv) If $w: A \vee B$ is in $\downarrow \Delta$, then $w: A$ and $w: B$ are in $\downarrow \Delta$.

(Lว) If $w: A \supset B$ is in $\downarrow \Gamma$, then either $w: A$ is in $\downarrow \Delta$ or $w: B$ is in $\downarrow \Gamma$.

(Rว) If $w: A \supset B$ is in $\downarrow \Delta$, then $w: A$ is in $\downarrow \Gamma$ and $w: B$ is in $\downarrow \Delta$

$\left(\mathrm{R} \Vdash^{\forall}\right.$ ) If $a \Vdash^{\forall} A$ is in $\downarrow \Delta$, then for some $w$ there is $w \in a$ in $\Gamma$ and $w: A$ in $\downarrow \Delta$.

$\left(\mathrm{LI}^{\forall}\right)$ If $w \in a$ and $a \Vdash^{\forall} A$ are in $\Gamma$, then $w: A$ is in $\downarrow \Gamma$.

$\left(\mathrm{R}^{\exists}{ }^{\exists}\right.$ ) If $w \in a$ is in $\Gamma$ and $a \Vdash^{\exists} A$ is in $\Delta$, then $w: A$ is in $\downarrow \Delta$.

$\left(\mathrm{L} \Vdash^{\exists}\right)$ If $a \Vdash^{\exists} A$ is in $\downarrow \Gamma$, then for some $w$ there is $w \in a$ in $\Gamma$ and $w: A$ is in $\downarrow \Gamma$.

(L口) If $w: \square A$ is in $\downarrow \Gamma$, then for some $a, a \in N(w)$ is in $\Gamma$ and $a \Vdash^{\forall} A$ is in $\downarrow \Gamma$.

(R口) If $a \in N(w)$ is in $\Gamma$ and $w: \square A$ is in $\downarrow \Delta$, then $a \Vdash^{\forall} A$ is in $\downarrow \Gamma$.

$(\mathrm{L} \diamond)$ If $a \in N(w)$ is in $\Gamma$ and $w: \diamond A$ is in $\downarrow \Gamma$, then $a \Vdash^{\exists} A$ is in $\downarrow \Gamma$.

$(\mathrm{R} \diamond)$ If $w: \diamond A$ is in $\downarrow \Delta$, then for some $a, a \in N(w)$ is in $\Gamma$ and $a \Vdash^{\exists} A$ is in $\downarrow \Delta$.

$(\mathrm{L} \forall)$ If $y \in D(w), w: \forall x A$ are in $\Gamma$, then $w: A[y / x]$ is in $\downarrow \Gamma$.

(R $\forall$ ) If $w: \forall x A$ is in $\downarrow \Delta$, then for some $z, z \in D(w)$ is in $\downarrow \Gamma$ and $w: A[z / x]$ in $\downarrow \Delta$.

(L $\exists$ ) If $w: \exists x A$ is in $\downarrow \Gamma$, then for some $z, z \in D(w)$ and $w: A[z / x]$ are in $\downarrow \Gamma$.

(R $\exists$ ) If $w: \exists x A$ is in $\Delta$ and $y \in D(w)$ is in $\Gamma$, then $w: A[y / x]$ is in $\downarrow \Delta$.

(Ref) If $x, w$ are in $\Gamma, \Delta$, then $w: x=x$ is in $\Gamma$.

(Repl) If $w: y=z, E[y / x]$ are in $\Gamma$, where $E$ is either $w: P$ or $x \in D(w)$, or $a(x) \in N(w)$, then $E[z / x]$ is in $\Gamma$.

(Rig) If $w: y=z$ is in $\Gamma$ and $v$ is in $\Gamma, \Delta$, then $v: y=z$ is in $\Gamma$.

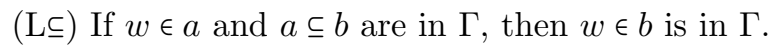

(Cons) If $x, w$ are in $\Gamma, \Delta$, then $x \in D(w)$ is in $\Gamma$.

(Prebasic) If $a \in N(w), b \in N(w)$ are in $\Gamma$, then for some $c, c \in N(w), c \subseteq a, c \subseteq b$ are in $\Gamma$.

(Nondeg) If $w$ is in $\Gamma, \Delta$, then for some $a, a \in N(w)$ is in $\Gamma$.

A branch is saturated relative to a system $\mathcal{S}$ of rules if it is saturated with respect to each rule of $\mathcal{S}$. 
Observe that in some cases of the above definition the downarrow $(\downarrow)$ does not appear: the reason is that it is not needed because the principal formula(s) is(are) copied in the premiss.

The definition of saturated branch is extended to infinite branches $\mathcal{B} \equiv\left\{\Gamma_{i} \Rightarrow \Delta_{i}\right\}_{i \geq 0}$ by replacing, in the definition above, $\Gamma$ (or $\downarrow \Gamma$ ) by $\Gamma$, the union of the $\Gamma_{i}$, and $\Delta$ (or $\downarrow \Delta$ ) by $\boldsymbol{\Delta}$, the union of the $\Delta_{i}$. The first clause (Init) is modified to requiring that for all $i$, there is no $w: P$ in $\Gamma_{i} \cap \Delta_{i}$.

Given a sequent $\Gamma \Rightarrow \Delta$ we apply root-first all the available rules. Observe that, by invertibility of the rules, there is no prescribed order in which they need to be applied. We want to avoid the possibility that the search produces an infinite branch which is not saturated, something that would result, e.g., from applying the same rule infinitely many times in consecutive steps. This is achieved as usual in such proofs through a counter: if there are $m$ rules, at step 1 we apply rule $R_{1}$ to all formulas that match its conclusion, at step 2 rule $R_{2}$, and in general for all $n \leq m$ we apply at step $n$ all possible instances of rule $R_{n}$. In this way we'll obtain a proof-search tree that can be either a derivation, or a non-derivation; the latter can either be a finite search tree that contains finite saturated branches, or an infinite search that, by König's lemma, contains an infinite saturated branch. We shall now prove that a saturated branch (either finite or infinite) for a sequent $\Gamma \Rightarrow \Delta$ gives a countermodel.

LEMMA 4.4

Let $\mathcal{B} \equiv\left\{\Gamma_{i} \Rightarrow \Delta_{i}\right\}$ be a branch in a proof-search tree for $\Gamma \Rightarrow \Delta$ saturated relative to a system $\mathcal{S}$ of rules. Then there exists a countermodel $\mathcal{M}$ to $\Gamma \Rightarrow \Delta$, which makes all the formulas in $\boldsymbol{\Gamma}$ true, and all the formulas in $\boldsymbol{\Delta}$ false. The countermodel is in the class of models relative to the logic Q.L that corresponds to the system $\mathcal{S}$ of rules.

Proof. Consider a saturated branch and define the countermodel $\mathcal{M} \equiv(W, \mathcal{N}, D, d, \mathcal{V})$ as follows:

1. The set $W$ of worlds consists of all the world labels in $\boldsymbol{\Gamma}$;

2. For each neighbourhood label $a$ in $\boldsymbol{\Gamma}$, we associate $\alpha_{a}$, the set that consists of all the $w$ in $W$ such that $w \in a$ is in $\boldsymbol{\Gamma}$;

3. For each $w$ in $W$, the set of neighbourhoods of $w$ consists of all the $\alpha_{a}$ such that $a \in N(w)$ is $\boldsymbol{\Gamma}$;

4. The set $D$ consists of all $x_{\equiv}$, where $x_{\equiv}$ is the equivalence class of all variables such that $v: x=y$ (for some $v$ ) occurs in $\boldsymbol{\Gamma}$ (it is immediate to see that $x_{\equiv}$ is an equivalence class since identity is an equivalence relation);

5 . For each $w$ in $W, d(w)$ consists of all the $x_{\equiv}$ such that $x \in D(w)$ is $\boldsymbol{\Gamma}$.

6. The valuation is defined on atomic formulas by $w \Vdash P$ if $w: P$ is in $\Gamma$ and is extended to arbitrary labelled formulas following the clauses of Definition 4.1.

We then define a realization $(\rho, \nu)$ by $\rho(w) \equiv w$ and $\nu(a) \equiv \alpha_{a}$. We define the assignment $\sigma$ as $\sigma(x) \equiv x_{\equiv}$. Next we prove the following:

1. If $A$ is in $\boldsymbol{\Gamma}$, then $\mathcal{M} \vDash_{\rho, \nu, \sigma} A$.

2. If $A$ is in $\boldsymbol{\Delta}$, then $\mathcal{M} \neq_{\rho, \nu, \sigma} A$.

The two claims are proved simultaneously by cases/induction on the weight of $A$ (cf. Definition 3.5). 


\section{Proof theory for quantified monotone modal logics}

(a) If $A$ is a formula of the form $a \in N(w), w \in a$, or $x \in D(w)$, claim 1 holds by definition of $\mathcal{M}$. Claim 2 is empty for these subcases because such formulas never occur on the right-hand side of sequents.

(b) If $A$ is a formula of the form $w: x=y$ and $a$ is in $\boldsymbol{\Gamma}$, we have $\sigma(x)=\sigma(y)$ because $x$ and $y$ are in the same equivalence class of variables, so $\mathcal{M} \vDash_{\rho, \nu, \sigma} w: x=y$. If $w: x=y$ is in $\boldsymbol{\Delta}$ then saturation clause Init entails that $x$ and $y$ are in different

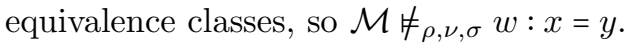

(c) If $A$ is a labelled atomic formula $w: P$, the claims hold by definition of $\Vdash$ and by the saturation clause Init no inconsistency arises. If $A$ is $\perp$, it holds by definition of the forcing relation that it is never forced, and therefore 2 holds, whereas 1 holds by the saturation clause for $L \perp$. If $A$ is a conjunction, or a disjunction, or an implication, the claim holds by the corresponding saturation clauses and inductive hypothesis on smaller formulas.

(d) If $a \Vdash^{\exists} A$ is in $\boldsymbol{\Gamma}$, by the saturation clause $\left(\mathrm{L}^{\exists}{ }^{\exists}\right)$, for some $w, w \in a$ and $w: A$ are in $\boldsymbol{\Gamma}$. Then $\mathcal{M} \vDash_{\rho, \nu, \sigma} w \in a$ by clause (a) above and by induction hypothesis $\mathcal{M} \vDash_{\rho, \nu, \sigma} w: A$, therefore $\mathcal{M} \vDash_{\rho, \nu, \sigma} a \Vdash^{\exists} A$.

If $a \Vdash^{\exists} A$ is in $\Delta$, then it is in $\Delta$ because such formulas are always copied to the premisses in the right-hand side of sequents. Consider an arbitrary world $w$ in $\alpha_{a}$. Then, by definition of $\mathcal{M}$ we have $w \in a$ in $\Gamma$ and thus, by the saturation clause $\left(\mathrm{R}{ }^{\exists}\right)$, we also have that $w: A$ is in $\boldsymbol{\Delta}$. By induction hypothesis we have $\mathcal{M} \neq_{\rho, \nu, \sigma} w: A$ and therefore $\mathcal{M} \neq_{\rho, \nu, \sigma} a \Vdash^{\exists} A$. The proof for formulas of the form $a \Vdash^{\forall} A$ is similar.

(e) If $w: \square A$ is in $\boldsymbol{\Gamma}$, then for some $a, a \in N(w)$ and $a \Vdash^{\forall} A$ are in $\boldsymbol{\Gamma}$. By induction hypothesis we obtain $\mathcal{M} \vDash_{\rho, \nu, \sigma} a \Vdash^{\forall} A$, and therefore $\mathcal{M} \vDash_{\rho, \nu, \sigma} w: \square A$.

If $w: \square A$ is in $\Delta$, then because of the form of the rules of the calculus it actually is in $\Delta$. Let $\alpha_{a}$ be a neighbourhood in $\mathcal{N}(w)$ in the model. By the saturation clause, we have that $a \Vdash^{\forall} A$ is in $\Delta$. By induction hypothesis we obtain $\mathcal{M} \sharp_{\rho, \nu, \sigma} a \Vdash^{\forall} A$, and therefore $\mathcal{M} \neq_{\rho, \nu, \sigma} w: \square A$.

(f) If $w: \diamond A$ is in $\boldsymbol{\Gamma}$, then it actually is in $\Gamma$. Assume that $\alpha_{a}$ is a neighbourhood in $\mathcal{N}(w)$, then $a \in N(w)$ is in $\Gamma$ and by saturation $a \Vdash^{\exists} A$ is in $\boldsymbol{\Gamma}$. By induction hypothesis we obtain $\mathcal{M} \vDash_{\rho, \nu, \sigma} a \Vdash^{\exists} A$ and therefore $\mathcal{M} \vDash_{\rho, \nu, \sigma} w: \diamond A$.

If $w: \diamond A$ is in $\boldsymbol{\Delta}$, then for some $a, a \in N(w)$ is in $\Gamma$ and $a \Vdash^{\exists} A$ is in $\boldsymbol{\Delta}$. By induction we have that $\mathcal{M} \neq_{\rho, \nu, \sigma} a \Vdash^{\exists} A$, and hence $\mathcal{M} \neq_{\rho, \nu, \sigma} w: \diamond A$.

(g) If $w: \forall x A$ is in $\boldsymbol{\Gamma}$, consider all the domain atoms $z \in D(w)$. If there is no such atom, then by definition, $\forall x A$ is forced at $w$ in the model. Else we find $w: A[z / x]$ in $\boldsymbol{\Gamma}$, forced in the model by the inductive hypothesis, and therefore $w: \forall x A$ is forced as well.

If $w: \forall x A$ is in $\boldsymbol{\Delta}$, we find by construction $z \in D(w)$ in $\boldsymbol{\Gamma}$ and $w: A[z / x]$ in $\boldsymbol{\Delta}$. By the inductive hypothesis, $A[z / x]$ is not forced at $w$ in the model, where $z$ is an element of the domain $d(w)$, thus $w: \forall x A$ is not forced either.

(h) The cases with $w: \exists x A$ in $\boldsymbol{\Gamma}$ and $w: \exists x A$ in $\boldsymbol{\Delta}$ are symmetric to those of $w: \forall x A$ in $\boldsymbol{\Delta}$ and $w: \forall x A$ in $\boldsymbol{\Gamma}$, respectively.

(i) In order to prove completeness for extensions of Q.M we need to prove that the countermodel $\mathcal{M}$ is in the intended class. If the branch is saturated with respect to Prebasic, observe that because of rule $L \subseteq$, if $c \subseteq a \in \boldsymbol{\Gamma}$, we have $\alpha_{c} \subseteq \alpha_{a}$, that is, extensional inclusion in the model respects formal inclusion. It therefore follows that the defined countermodel is pre-basic.

If we have saturation with respect to Nondeg, then for every world $w$ in the model, 
$\mathcal{N}(w)$ is non-empty, so the countermodel is non-degenerate.

Finally, if we have saturation with respect to rule Cons, for every world $w$ in $W$ and for every object $x_{\equiv}$ in $\mathrm{D}$, the formula $x \in D(w)$ is in $\boldsymbol{\Gamma}$, so the countermodel has constant domain.

We are ready to prove the completeness of the calculi.

THEOREM 4.5

If $A$ is valid (respectively, valid over all non-degenerate/pre-basic/ constant-domain frames) then there is a derivation in G3Q. $\mathbf{M}^{*}$ (in G3Q. $\mathbf{M}^{*}+\{$ Nondeg/Prebasic/Cons $\}$ ) of $\Rightarrow w: A$, for any label $w$.

Proof. For every $A$ we either find a derivation or a saturated branch. By the above lemma a saturated branch gives a countermodel to $A$. It is also immediate to notice that saturation under rule Nondeg (Prebasic, Cons) ensures that the countermodel is based on a frame that is non-degenerate (pre-basic, with constant domain, respectively). It follows that if $A$ is valid it has to be derivable.

\section{$5 \quad$ Necessity of Identity and Barcan Formulas}

In this section, we shall establish which rules are needed to derive in calculi based on G3Q.M* the Necessity of Identity

$$
x=y \supset \square x=y
$$

and the Barcan Formulas

$$
\begin{aligned}
& \square \forall x A \supset \forall x \square A \\
& \forall x \square A \supset \square \forall x A
\end{aligned}
$$

This will be done by applying a sort of 'bootstrapping' procedure: we shall use the rules of the basic calculus G3Q.M to find the other rules that are needed, in a rootfirst proof search, to derive such formulas. We shall rely on the structural results already established for G3Q.M* , especially invertibility of all its rules. On the other hand, in discussing the outcome of this procedure, we shall assume the soundness and completeness results - to be proved in Section 5 - in order to transform the rules we found into frame properties.

We begin with $N I$; rule Nondeg, needed to derive the Necessity of Identity, is in Table 2 .

Proposition 5.1

$$
\begin{aligned}
& \text { G3Q.M* } \left.{ }^{*} \text { Nondeg }\right\} \vdash N I \\
& \qquad \frac{v: x=y, v \in a, a \in N(w), w: x=y \Rightarrow w: \square x=y, v: x=y}{\frac{v \in a, a \in N(w), w: x=y \Rightarrow w: \square x=y, v: x=y}{a \in N(w), w: x=y \Rightarrow w: \square x=y, a \Vdash^{\forall} x=y}} R \Vdash^{\forall} \\
& \frac{a \in N(w), w: x=y \Rightarrow w: \square x=y}{\frac{w: x=y \Rightarrow w: \square x=y}{2}} \text { Nondeg }
\end{aligned}
$$


22 Proof theory for quantified monotone modal logics

TABLE 4: Rules for CBF

$\frac{b \in N(w), b \subseteq a, \operatorname{In}(b, w), a \in N(w), \Gamma \Rightarrow \Delta}{a \in N(w), \Gamma \Rightarrow \Delta}$ SubIn, bfresh $\quad \frac{y \in D(v), v \in b, \operatorname{In}(b, w), y \in D(w), \Gamma \Rightarrow \Delta}{v \in b, \operatorname{In}(b, w), y \in D(w), \Gamma \Rightarrow \Delta}$ In

It is immediate to see that the same rule Nondeg is needed to derive the Necessity of Distinctness: $N D:=x \neq y \supset \square x \neq y$. Moreover, rule Nondeg is also needed to derive the necessitation axiom $N$ : $\square$ T in G3Q.M*. Thus, as expected, we found a strong relationship between $N I, N D$ and $N$ in QMML: each of them is valid over the class of all monotone neighbourhood frames where for all $w \in W, \mathcal{N}(w) \neq \varnothing$.

Now we move to the Converse Barcan Formula $C B F$. The proof search tree for $C B F$ in the derivation below invokes rules that are listed in Table 4:

Proposition 5.2

G3Q. $\mathbf{M}^{*}+\{S u b I n, I n\} \vdash C B F$

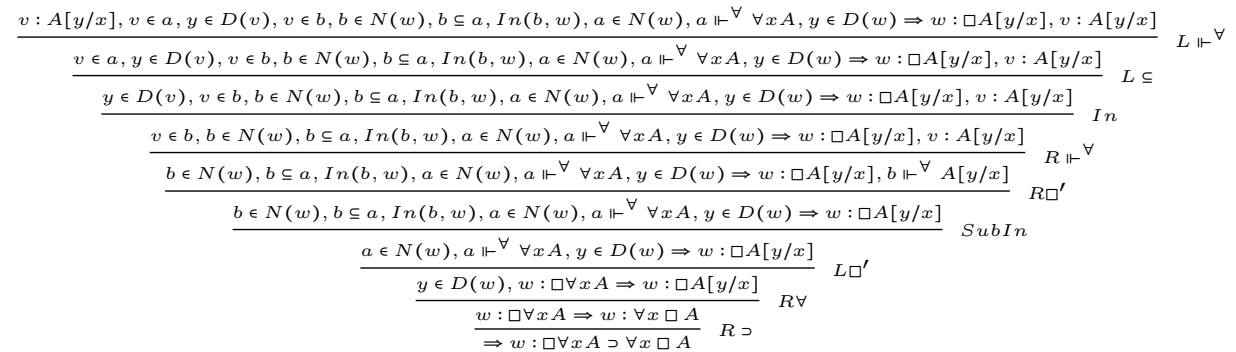

Rule SubIn requires that each neighbourhood $a$ of a state $w$ contains another neighbourhood $b$ of $w$ with a certain property $\operatorname{In}(b, w)$ specified in the rule In, namely that the inner domain of each member of $b$ is an extension of that of $w$. This shows that in a varying domain neighbourhood semantics, $C B F$ is not valid over all monotone frames, as it happens over constant domain neighbourhood frames, cf. Theorem 6.1. The difference is that frames have also to satisfy a property generalizing that for increasing domains in normal modal logics. Notice that as a limit case we can consider a rule analogous to the one for increasing domains in calculi for normal modal logics:

$$
\frac{y \in D(v), y \in D(w), v \in a, a \in N(w), \Gamma \Rightarrow \Delta}{y \in D(w), v \in a, a \in N(w), \Gamma \Rightarrow \Delta} \text { Incr }
$$

In G3Q. $\mathbf{M}^{*}+\{$ Incr $\}$ we have the following simpler derivation of $C B F$ :

$$
\begin{aligned}
& \underline{y \in D(v), v \in a, a \in N(w), v: \forall x A, v: A[y / x], a \Vdash^{\forall} \forall x A, y \in D(w) \Rightarrow w: \square A[y / x], v: A[y / x]} L \forall \\
& \underline{y \in D(v), v \in a, a \in N(w), v: \forall x A, a \Vdash^{\forall} \forall x A, y \in D(w) \Rightarrow w: \square A[y / x], v: A[y / x]} \text { Incr } \\
& \underline{v \in a, a \in N(w), v: \forall x A, a \Vdash^{\forall} \forall x A, y \in D(w) \Rightarrow w: \square A[y / x], v: A[y / x]} L \Vdash^{\forall} \\
& \underline{v \in a, a \in N(w), a \Vdash^{\forall} \forall x A, y \in D(w) \Rightarrow w: \square A[y / x], v: A[y / x]} R \Vdash^{\forall} \\
& \underline{a \in N(w), a \Vdash^{\forall} \forall x A, y \in D(w) \Rightarrow w: \square A[y / x], a \Vdash^{\forall} A[y / x]} R \square^{\prime} \\
& \underline{a \in N(w), a \Vdash^{\forall} \forall x A, y \in D(w) \Rightarrow w: \square A[y / x]} L \square^{\prime} \\
& \underline{y \in D(w), w: \square \forall x A \Rightarrow w: \square A[y / x]} R \forall \\
& \frac{w: \square \forall x A \Rightarrow w: \forall x \square A}{\Rightarrow w: \square \forall x A \supset \forall x \square A} R \supset
\end{aligned}
$$


TABLE 5: Rules for BF

\begin{tabular}{ll}
\hline \hline$\frac{b \in N(w), \operatorname{Min}(b, a, w), a \in N(w), \Gamma \Rightarrow \Delta}{a \in N(w), \Gamma \Rightarrow \Delta} \operatorname{Min}, b$ fresh & $\frac{y \in D(w), v \in b, \operatorname{Min}(b, a, w), y \in D(v), \Gamma \Rightarrow \Delta}{v \in b, \operatorname{Min}(b, a, w), y \in D(v), \Gamma \Rightarrow \Delta} \operatorname{Min} D$ \\
$\frac{v \in a(y), v \in b, \operatorname{Min}(b, a, w), a(y) \in N(w), y \in D(w), \Gamma \Rightarrow \Delta}{v \in b, \operatorname{Min}(b, a, w), a(y) \in N(w), y \in D(w), \Gamma \Rightarrow \Delta} \operatorname{MinN}$ & $\frac{y \in D(w), \Gamma \Rightarrow \Delta a \in N(w), \Gamma \Rightarrow \Delta}{\Gamma \Rightarrow \Delta}$ NE, y, afresh \\
\hline \hline
\end{tabular}

Nevertheless, Proposition 5.2 shows that rule Incr is not necessary to derive $C B F$ since it is enough to consider frames whose domains are increasing with respect to some subset of a given neighbourhood.

Lastly, we consider the Barcan Formula BF. The proof search tree is in Proposition 5.3 and the rules we found are in Table 5 .

\section{Proposition 5.3

$$
\text { G3Q.M* }{ }^{*}+\{\operatorname{Min}, \operatorname{Min} D, \operatorname{MinN}, N E\} \vdash B F
$$

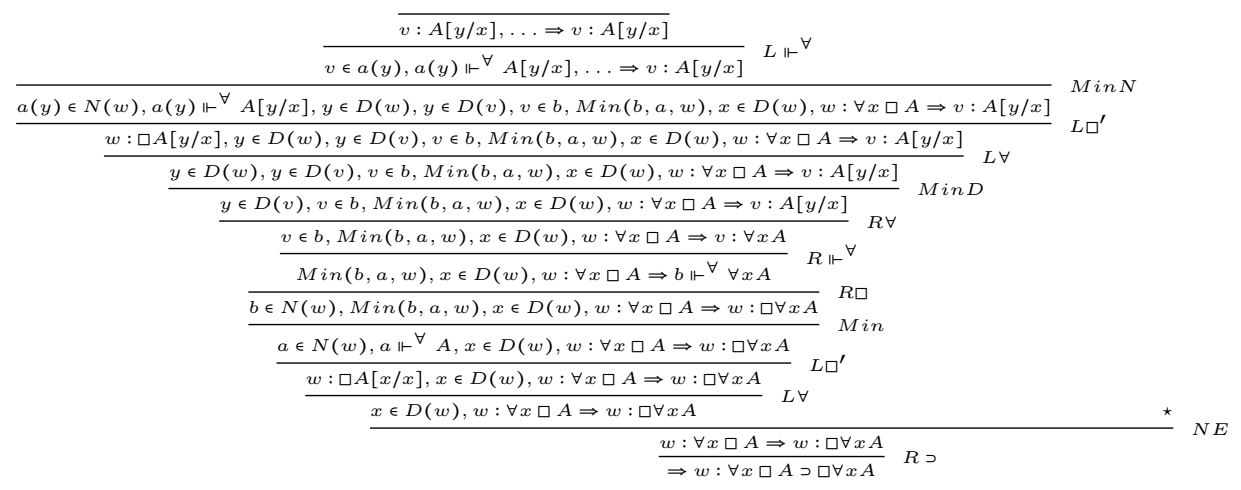

The right branch $\star$ above $N E$ is like the left one, but without the lowermost instances of $L \forall$ and $L \square^{\prime}$. In the left branch these two rule instances are needed only to introduce (bottom-up) $a \in N(w)$ which, in the right branch, is introduced directly by $N E$.

Roughly, rule $N E$ ensures that no world is such that both its inner domain and its set of neighbourhoods are empty. The rules Min, MinN and MinD are more complicated and require a more detailed explanation. Essentially their role is the following: they ensure the existence of a minimal neighbourhood for every family of neighbourhoods that are indexed by elements of the domain. This minimal neighbourhood $b$, whose existence is granted by rule Min, respects a decreasing-domain-like property in that each object in the inner domain of one member of $b$ is also in the inner domain of the base state. Moreover, thanks to rule $\operatorname{MinN}, b$ is minimal in the sense that it is contained in each neighbourhood of the base world that is part of a family of neighbourhoods indexed by objects in its inner domain. This explains the need for functional labels: they are needed to keep track of the dependency of neighbourhoods on existing objects when we consider minimal neighbourhoods that are needed to derive $B F$. Observe that rule $M i n N$ is similar to rule Prebasic in Table 2, which is needed to derive $C$ in calculi for monotone modalities, but it is independent of it because it gives us a subset of a set $a_{1}, \ldots a_{n}$ of neighbourhoods of a state $w$ only 


\section{Proof theory for quantified monotone modal logics}

if each $a_{i}$ is a member of a family of neighbourhoods that is indexed by a different object of the inner domain of $w$. Therefore, in general $B F$ and $C$ are independent of each other over varying domain neighbourhood frames.

\section{A digression on the Barcan Formula}

The Barcan Formula is a very strong property, as shown by its paradoxical consequences when it involves a modality with a non-normal interpretation, as in the lottery paradox. It is therefore not surprising that its derivation in our system should require additional strong properties on the neighbourhood semantics. Here we have shown that the existence of minimal neighbourhoods for families of neighbourhoods indexed by elements of the domain is a sufficient condition for the validity of the Barcan Formula.

This minimality property can be related to the characterizing property of Alexandrov topologies, namely the fact that open sets are closed under arbitrary intersections. There are several equivalent characterizations of Alexandrov topologies, among these the property that the intersection of neighbourhoods ${ }^{5}$ of a point is still a neighbourhood of the point and the property that every point has a smallest neighbourhood. The most interesting consequence of being an Alexandrov topology is something that bridges topology in the most traditional sense of mathematical analysis to applications in ordered structures. We briefly recall the essential part of the link. ${ }^{6}$

Given a topological space $(X, \tau)$ we can define a preorder (called the specialization preorder) by

$$
x \leqslant{ }^{\tau} y \equiv \forall a \in \tau(x \in a \supset y \in a)
$$

Conversely, given a preorder $(X, \leqslant)$ we can define a topology $\tau \leqslant$ by taking as opens the subsets of $X$ which are upper sets with respect to $\leqslant$, i.e. subsets $a$ of $X$ with the property $\forall x, y(x \in a \wedge x \leqslant y \supset y \in a)$. We indicate the property of $a$ being an upper set with $U(a)$. It is then natural to ask whether these two operations between topological spaces and preorders are inverse of each other. It is easy to verify that $x \leqslant \tau \leqslant y$ if and only if $\forall a \in \tau \leqslant(x \in a \supset y \in a)$ if and only if $\forall a(U(a) \wedge x \in a \supset y \in a)$ if and only if $x \leqslant y$. However, in general the topology $\tau^{\leqslant}$is a finer topology than $\tau$, i.e. it has more open sets: If $a$ is $\tau$, it is immediate to see that it is an upper set with respect to the specialization preorder, i.e. $a$ is in $\tau^{\leqslant \tau}$. We show that the converse holds when the topology is Alexandrov. So, let $a$ be in $\tau^{{ }^{\tau}}$; to prove that $a$ is actually in $\tau$ we need to prove that for every $x \in a$ there is a neighbourhood of $x$ included in $a$. Let $c$ be the intersections of all open sets in $\tau$ that contain $x$. By the Alexandrov property, $c$ is in $\tau$, so we only have to prove that $c$ is included in $a$. Let $y \in c$, i.e., by definition of $c, \forall b \in \tau(x \in b \supset y \in b)$, i.e. $x \leqslant{ }^{\tau} y$. Since $x$ is in $a$ and $a$ is an upper set w.r.t. $\leqslant^{\tau}$, we have $y \in a$. Since $y$ was arbitrary in $c, c \subseteq a$.

The equivalence between Alexandrov topologies and topologies induced by a preorder is known also in the context of possible worlds semantics as the equivalence between relational models and neighbourhood models with the property of monotonicity (upward closure) and the property that the intersection of the neighbourhoods of $w$ is

\footnotetext{
${ }^{5}$ Recall that a neighbourhood of a point $x$ in a topological space $(X, \tau)$ is a subset of $X$ that contains an open of $\tau$ that contains $x$, and an open set is a subset of $X$ with the property that for every point $x$ it contains, there is a neighbourhood of $x$ included in it.

${ }^{6}$ For a reference to this correspondence see [11], p. 45; observe however that Johnstone requires the $T_{0}$ property to get an order (with antisymmetry) rather than just a preorder and the details are left as exercises.
} 
a neighbourhood of $w$. Since relational models characterize normal modal logics, it is interesting to ask ourselves whether imposing validity of the Barcan Formula takes to a collapse of neighbourhood semantics into relational semantics, and therefore makes the logic normal.

We have seen that to get validity of the Barcan Formula it is enough to require the existence of minimal neighbourhoods (w.l.o.g., intersections) only for families indexed by elements of the domain. So the question reduces to asking whether arbitrary families of neighbourhoods can be indexed by elements of the domain. Clearly, the cardinality of a family of neighbourhoods of $w$ is bounded by the number of elements in $\mathcal{N}(w)$, which in turn is bounded by the cardinality of the power set of $W, \mathcal{P}(W)$. Thus in the end, an indexed family of neighbourhoods of $w$ can be reduced to a family indexed by the domain of $w$ if the cardinality of the domain is at least the cardinality of $\mathcal{P}(W)$.

\section{Structural properties}

The proof of all the structural properties of the afore-mentioned extensions is a routine exercise and we shall not give it in detail, but just observe that, because of the form of the rules, it proceeds exactly as in Section 3.4.

\section{Soundness and completeness}

In order to extend the soundness and completeness results to QMML containing the Necessity of Identity and/or the Barcan Formulas, we start by defining classes of monotone neighbourhood frames where such formulas are valid.

DEFINITION 5.4 (Frame properties)

Let $\mathcal{F}$ be a varying domain (monotone) neighbourhood frame. We say that $\mathcal{F}$ is a frame for

- $N I$ whenever $\mathcal{F}$ is non-degenerate: for all $w \in \mathcal{W}, \mathcal{N}(w) \neq \varnothing$;

- $C B F$ whenever $\mathcal{F}$ is subset-increasing: for each $w \in \mathcal{W}$ and each $a \in \mathcal{N}(w)$ there is $b \in \mathcal{N}(w)$ such that:

1. $b \subseteq a$;

2. For all $v \in b, o \in d(w)$ implies $o \in d(v)$.

- $B F$ whenever $\mathcal{F}$ is minimal-decreasing: for each $w \in W$

1. $d(w) \cup \mathcal{N}(w) \neq \varnothing$;

2. If $\mathcal{I} \subseteq d(w)$, then there is $b \in \mathcal{N}(w)$ such that:

(a) If $i \in \mathcal{I}$ and $a(i) \in \mathcal{N}(w)$, then $b \subseteq a(i)$, and

(b) If $v \in b$ and $o \in d(v)$, then $o \in d(w)$.

TheOREM 5.5 (Soundness)

If $\Gamma \Rightarrow \Delta$ is derivable in G3Q. $\mathbf{M}^{*}+\{$ Nondeg $\}$, G3Q. $\mathbf{M}^{*}$

$+\{\operatorname{SubIn}, \operatorname{In}\}$, or G3Q.M $\mathbf{M}^{*}+\{\operatorname{Min}, \operatorname{MinD}, \operatorname{MinN}, N E\}$, then it is valid, respectively, in the class of all quantified monotone neighbourhood frames for $N I, C B F$, or $B F$.

Proof. The proof extends that of Theorem 4.2 by considering the new inductive cases given by the non-logical rules for $N I, C B F$ and $B F$. 


\section{Proof theory for quantified monotone modal logics}

It is easy to see that these rules preserve validity over the appropriate class of frames. To illustrate, we consider rule SubIn. Assume the premiss valid and consider an arbitrary $S N$-realization $(\rho, \nu)$ and an arbitrary assignment $\sigma$ such that $\mathcal{M} \vDash_{\rho, \nu, \sigma}$ $a \in N(w), \Gamma$ with $\mathcal{M}$ based on a subset-increasing frame. This means that, for some fresh label $b, \nu(b)$ is such that $\nu(b) \subseteq \nu(a), \nu(b) \in \mathcal{N}(w)$, and for all $v$ such that $\rho(v) \in \nu(b)$, and all $x$ such that $\sigma(x) \in d(\rho(w))$, we have that $\sigma(x) \in d(\rho(v))$. This latter fact implies that $\mathcal{M}_{\rho, \nu, \sigma} \vDash \operatorname{In}(b, w)$, and, since $\mathcal{M}_{\rho, \nu, \sigma} \vDash b \in N(w), b \subseteq a$, by induction hypothesis we conclude that there is $G \in \Delta$ such that $\mathcal{M} \vDash_{\rho, \nu, \sigma} G$.

Theorem 5.6 (Completeness)

If $A$ is valid in the class of all quantified monotone neighbourhood frames for $N I$, $C B F$, or $B F$, then $\Rightarrow w: A$ is derivable in G3Q. $\mathbf{M}^{*}+\{N o n d e g\}, \mathbf{G} 3 \mathbf{Q} \cdot \mathbf{M}^{*}+\{\operatorname{SubIn}, \operatorname{In}\}$, or G3Q.M ${ }^{*}+\{\operatorname{Min}, \operatorname{MinD}, \operatorname{MinN}, N e\}$.

Proof. The proof extends that of Theorem 4.5. The notion of saturation, Definition 4.3 , and the truth lemma, 4.4, are extended to cover the new rules. The details are left to the reader.

We have only to prove that the countermodel $\mathcal{M}$ given by a saturated branch is based of a frame for $N I, C B F$, or $B F$, respectively. To illustrate, if we are in G3Q. $\mathbf{M}^{*}+\{\operatorname{Min}, \operatorname{Min} D, \operatorname{MinN}, N E\}$, we have to prove that $\mathcal{M}$ is based on a minimaldecreasing frame. Let us consider a generic $w \in W$. By saturation under rule $R E$ we immediately have that $d(w) \cup \mathcal{N}(w) \neq \varnothing$. Now, take $\mathcal{I} \subseteq d(w)$ such that, for each $i \in \mathcal{I}$, the neighbourhood $a(i) \in \mathcal{N}(w)$ and, therefore, the formula $a(i) \in N(w)$ is in $\boldsymbol{\Gamma}$. By saturation under rule Min, there is a label $b$ such that both $b \in N(w)$ and $\operatorname{Min}(b, a, w)$ are in $\boldsymbol{\Gamma}$. This already ensures that $\alpha_{b} \in \mathcal{N}(w)$. Moreover, by saturation under rule $\operatorname{MinN}$ and by construction of $\mathcal{M}$, we have that $v \in \alpha_{b}$ implies $v \in \alpha_{a(i)}$, for each $i \in \mathcal{I}$. Finally, by saturation under rule $\operatorname{Min} D$ and by construction of $\mathcal{M}$, we have that $v \in \alpha_{b}$ and $i \in d(v)$ imply $i \in d(w)$. We conclude that $\mathcal{M}$ is based on a minimal-decreasing frame.

\section{Related work and conclusion}

The only kind of proof systems that have been presented in the literature for QNML are axiomatic systems, studied in $[1,2,4,20,21]$. We sketch here the main results that have been obtained in [2] for logics with constant domain and those in [4] for logics with varying domain.

In [2] a language without identity and a constant domain neighbourhood semantics is considered. The following correspondence results are proved for $C B F$ and $B F$ over constant domain frames:

Theorem $6.1([2])$

1. The Converse Barcan Formula $C B F$ is valid over constant domain neighbourhood frames that are either monotone or such that $|D|=1$.

2. The Barcan Formula $B F$ is valid over constant domain neighbourhood frames that

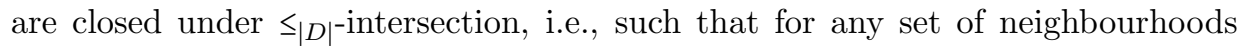
$\{a(i): i \in \mathcal{I}\}$, if $|\mathcal{I}| \subseteq|D|$ and, for all $i \in \mathcal{I}, a(i) \in \mathcal{N}(w)$, then $\bigcap_{i \in \mathcal{I}} a(i) \in \mathcal{N}(w)$. 
This theorem shows that over constant domain frames there is a very strong relation between $C B F$ and $M$ as well as between $B F$ and $C$ : if the frame has a domain containing more than one object (it is nontrivial), then $C B F$ and $M$ are valid over the same class of frames, and, provided the domain is finite, the same holds for $B F$ and $C$. As it is shown in [2], if the domain is infinite, it is possible to construct a countermodel for $B F$ over a frame validating $C$.

The proof systems for quantified non-normal modal logics considered in [2] are axiomatic systems. Given an axiomatic presentation of the propositional non-normal modal logic $\mathbf{E}(\mathbf{M})$, Costa and Pacuit [2, Theorems 3.20 and 3.21] consider its quantified extension Q.E(M) and prove that $\mathbf{Q . E}(\mathbf{M})$ is sound and complete with respect to the class of all constant domain (monotone) neighbourhood frames. It is important to notice that soundness and completeness for a generic logic Q.L, possibly containing $C B F$ and/or $B F$, are proved not with respect to (classes of) constant domain frames, but with respect to classes of constant domain general frames, i.e. a frame together with a well-behaved restriction on the set of possible valuations over it.

If we limit ourselves to monotone constant domain frames, the results in Theorem 6.1.1 can be simplified in such a way that the class of constant domain frames considered becomes the one we have considered in Definition 5.4 for $C B F$ and over which we have proved that the appropriate labelled calculus is sound and complete. It follows that the labelled calculus G3Q. $\mathbf{M}^{\star}+$ Cons $(+S u b I n, I n)$ and the axiomatic system $\mathbf{Q} \cdot \mathbf{M}^{\star}(+C B F)$ allow to prove the same identity-free $\mathcal{L}^{\mathcal{S}}$-formulas.

In [4], quantified non-normal logics with free quantification and identity are considered, but instead of a varying domain neighbourhood semantics, the paper considers a varying domain multirelational semantics. This semantics is a generalization of (varying domain) Kripke semantics for quantified normal modal logics where, instead of a single accessibility relation, we have a non-empty set of accessibility relations $\mathcal{R}=\left\{R_{i}: i \in \mathcal{I}\right\}$.

More precisely, a multirelational frame is a tuple: ${ }^{7}$

$$
\mathscr{F}=<W, \mathcal{R}, D, d,>
$$

where $W, D, d$, are as in Definition 3.2, and $\mathcal{R}$ is a non-empty set of binary accessibility relations $R_{i} \subseteq W \times W$. A multirelational model $\mathscr{M}$ is a multirelational frame augmented with a valuation $\mathcal{V}$ (cf. Definition 3.3); assigments are mappings $\sigma$ from the variables to the outer domain of $\mathscr{F}$. The satisfaction clauses are like the ones for neighbourhood models, save for the modalities, for which we have:

$$
\begin{array}{ccc}
\mathscr{M}, \sigma, w \Vdash \square A & \text { iff } & \exists R_{i} \in \mathcal{R}, R_{i}(w) \subseteq \llbracket A \rrbracket \mathscr{M}_{, \sigma} \\
\mathscr{M}, \sigma, w \Vdash \diamond A \quad \text { iff } \quad \forall R_{i} \in \mathcal{R}, R_{i}(w) \cap \llbracket A \rrbracket_{\mathscr{M}, \sigma} \neq \varnothing
\end{array}
$$

where $R_{i}(w) \equiv\left\{v \mid v \in W\right.$ and $\left.w R_{i} v\right\}$. The notions of truth in a model and of validity are defined as expected. There is a precise correspondence between multirelational frames and monotone non-degenerate neighbourhood frames, as it is shown by the following truth-preserving translation.

DEFINITION 6.2 (Translation between multirelational and non-degenerate neighbourhood frames)

\footnotetext{
${ }^{7}$ For simplicity, we consider multirelational frames where the outer domain $D$ is constant, and not increasing as in $[4]$.
} 
28 Proof theory for quantified monotone modal logics

1. Given a multirelational model $\mathscr{M}=\langle W, \mathcal{R}, D, d, \mathcal{V}\rangle$, the (monotone and nondegenerate) neighbourhood model $\mathcal{M}^{\mathscr{M}}=\left\langle W, \mathcal{N}^{\mathscr{M}}, D, d, \mathcal{V}>\right.$ is given by defining $\mathcal{N}^{\mathscr{M}}$ as the family of sets, for all $w \in W, \mathcal{N}^{\mathscr{M}}(w)$ where $a_{i} \in \mathcal{N}^{\mathscr{M}}(w)$ iff $a_{i} \supseteq R_{i}(w)$ (for $\left.R_{i} \in \mathcal{R}\right)$.

2. Given a monotone and non-degenerate neighbourhood model $\mathcal{M}=\langle W, \mathcal{N}, D, d, \mathcal{V}\rangle$, the multirelational model $\mathscr{M}^{\mathcal{M}}=<W, \mathcal{R}^{\mathcal{M}}, D, d, \mathcal{V}>$ is given by $\mathcal{R}^{\mathcal{M}} \equiv\left\{R_{a} \mid a \in \mathcal{N}\right\}$ where each $R_{a}$ is such that for all $w, v \in W, w R_{a} v$ iff $a \in \mathcal{N}(w)$ and $v \in a$.

LEMMA 6.3

1. Given a multirelational model $\mathscr{M}=\langle W, \mathcal{R}, D, d, \mathcal{V}\rangle$ and an $\mathcal{L}^{\mathcal{S}}$-formula $A$, we have: ${ }^{8}$

$$
\mathscr{M}, \sigma, w \Vdash A \quad \text { iff } \quad \mathcal{M}^{\mathscr{M}}, \sigma, w \Vdash A
$$

2. Given a monotone non-degenerate neighbourhood model $\mathcal{M}=\langle W, \mathcal{N}, D, d, \mathcal{V}\rangle$ and an $\mathcal{L}^{\mathcal{S}}$-formula $A$, we have:

$$
\mathcal{M}, \sigma, w \Vdash A \quad \text { iff } \quad \mathscr{M}^{\mathcal{M}}, \sigma, w \Vdash A
$$

Proof. Both proofs are by induction on the weight of $A$. The only non trivial cases are those where a modal operator is principal in $A$. For example, the case of 2 where $A \equiv \square B$ is as follows:

$$
\begin{array}{ll}
\mathcal{M}, \sigma, w \Vdash \square B & \text { iff } \\
\exists a \in \mathcal{N}(w), a \subseteq \llbracket B \rrbracket_{\mathcal{M}, \sigma} & \text { iff } \\
\exists a \in \mathcal{N}(w), \forall v \in W(v \in a \supset \mathcal{M}, \sigma, w \Vdash B) & \text { iff (by IH) } \\
\exists a \in \mathcal{N}(w), \forall v \in W\left(v \in a \supset \mathscr{M}^{\mathcal{M}}, \sigma, w \Vdash B\right) & \text { iff } \\
\exists R_{a} \in \mathscr{R}^{\mathcal{M}}, R_{a}(w) \subseteq \llbracket B \rrbracket_{\mathscr{M}^{\mathcal{M}}, \sigma} & \text { iff } \\
\mathscr{M}^{\mathcal{M}}, \sigma, w \Vdash \square B &
\end{array}
$$

It is immediate to notice that multirelational semantics validates the non-normal modal logic MN. Moreover, it validates the necessity of identity and of distinctness. For the Barcan Formulas, the following correspondence results are proven in [4]:

THEOREM $6.4([4])$

1. The Converse Barcan Formula $C B F$ is valid over all multirelational frames $\mathscr{F}$ such that:

$\forall w \in W$, if $\forall R_{k} \in \mathcal{R}, R_{k}(w) \neq \varnothing$, then $\forall R_{j} \in \mathcal{R}$ there is $R_{i} \in \mathcal{R}$ such that: (i) $R_{i}(w) \subseteq R_{j}(w)$ and (ii) $\forall v \in R_{i}(w), d(w) \subseteq d(v)$.

2. The Barcan Formula $B F$ is valid over all multirelational frames such that:

$\forall w \in W$, either $\exists R_{i} \in \mathcal{R}$ s.t. $R_{i}(w)=\varnothing$ or, if $n=|\mathcal{R}|$ and $m=\left|d_{w}\right|$ (possibly $\omega)$, then for any sequence of worlds $v_{1}, \ldots, v_{n}$ such that $w R_{i} v_{i}(i \leq n)$ and for any sequence of accessiblity relations $R_{j_{1}}, \ldots, R_{j_{m}}$, there is a world $u \epsilon$ $\left\{z_{1}, \ldots, z_{m}\right\} \cap \bigcap_{i \leq m} R_{j_{i}}(w)$ such that $d(w) \supseteq d(u)$.

${ }^{8}$ Without loss of generality, we do not consider $S N$-realizations. 
The proof systems for QMML considered in [4] are axiomatic systems. Given an axiomatic presentation of the monotone logic $\mathbf{E M N}$, let $\mathbf{Q}_{=}^{0} \cdot \mathbf{M N}(+C B F+\square E)$ be its extension to the quantified case obtained by adding axioms and rules for free quantification and the axioms for identity (and $C B F$ and the schema $\square E$ ), cf. [4] for the details. In [4, Theorems 6.11 and 6.13] it is proved that $\mathbf{Q}_{=}^{0} \cdot \mathbf{M N}$ and $\mathbf{Q}_{=}^{0} \cdot \mathbf{M N}+C B F+\square E$ are sound and complete (with respect to the appropriate classes of multirelational frames).

\section{Conclusion}

This paper has introduced labelled calculi for quantified monotone modal logics. In particular, we have given labelled calculi for both varying and constant domain monotone neighbourhood frames and we have considered the Barcan Formulas and the Necessity of Identity. We have shown that these calculi have good structural properties and that each calculus is sound and complete with respect to the appropriate class of quantified monotone neighbourhood frames. This means that our labelled calculi are equivalent (with respect to derivable formulas) to the axiomatic systems for monotone logics possibly containing $C B F$ considered in $[2,4]$. To our knowledge, we provided the first completeness result with respect to classes of neighbourhood frames validating $B F$. One of the main advantages of using labelled calculi is the possibility to give a uniform and modular completeness theorem that covers QMML with or without $N I, C B F$, and $B F$.

We have not considered labelled calculi for quantified non-monotone modal logics. For logics without $B F$ the extension to non-monotonic logics should be immediate. Nevertheless, $B F$ would become less tractable because over non-monotonic logics it requires closure under arbitrary intersection, and it is hard to capture this notion with finitary rules. We leave this problem for future research.

Notice that the bootstraping procedure used in Section 5 gives rules, and consequently semantic conditions, that are sufficient to derive (validate) the Necessity of Identity and the Barcan Formulas, but it does not show their optimality. With respect to constant domain monotone frames, their optimality (over non-trivial frames) follows from Theorem 6.1. With respect to (calculi for) varying domain frames, the optimality of the conditions we found for $N I$ and for $C B F$ follows from Theorem 6.4. We conjecture that the rules in Table 5 for $B F$ over (calculi for) varying domain neighbourhood frames are optimal, and we leave the proof for future research.

Another open direction for future research is the identification of decidable fragments of quantified monotone modal logics.

\section{Funding}

This work was partially supported by the Academy of Finland [research project no. 1308664].

\section{Acknowledjments}

Part of this research was completed when the authors were visiting the Hausdorff Research Institute for Mathematics at the University of Bonn during the Trimester 


\section{Proof theory for quantified monotone modal logics}

Program 'Types, Sets and Constructions'. Thanks are due to two anonymous referees and to audience at the EICNCL 2018 workshop.

\section{References}

[1] H. Arló-Costa. First order extensions of classical systems of modal logic. Studia Logica, 71, 87-118, 2002.

[2] H. Arló-Costa and E. Pacuit. First-order classical modal logic. Studia Logica, 84, 171-210, 2006.

[3] H. Arló Costa and E. Pacuit. Free quantified (classical) modal logic: Varying domains. Typescript CMU and ILLC-Amsterdam, 2006.

[4] E. Calardo and A. Rotolo. Quantification in some non-normal modal logics. Journal of Philosophical Logic, 46, 541-576, 2017.

[5] B.F. Chellas. Modal Logic: An Introduction. Cambridge University Press, 1980.

[6] R. Dyckhoff and S. Negri. Geometrization of first-order logic. The Bulletin of Symbolic Logic, 21, 123-163, 2015.

[7] M. Fitting. Modal proof theory. In Handbook of Modal Logic, P. Blackburn et al., eds, pp. 85-138. Elsevier, 2007.

[8] D.R. Gilbert and P. Maffezioli. Modular sequent calculi for classical modal logics. Studia Logica, 103, 175-217, 2015.

[9] G. Governatori and E. Luppi. Labelled tableaux for non-normal logics. In $A I^{*} I A$ 1999: Advances in Artificial Intelligence, LNCS vol. 1792, E. Lamma and P. Mello, eds, pp. 119-130. Springer, 2000.

[10] H. E. Kyburg and C.M. Teng. The logic of risky knowledge. Electronic Notes in Theoretical Computer Science, 67, 254-262, 2002.

[11] P.T. Johnstone. Stone Spaces, Cambridge University Press, 1982.

[12] S. Negri. Proof analysis in modal logic. Journal of Philosophical Logic, 34, 507-544, 2005.

[13] S. Negri. Proof theory for non-normal modal logics: The neighbourhood formalism and basic results. IfCoLog Journal of Logics and their Applications, 4, 241-1286, 2017.

[14] S. Negri and N. Olivetti. A sequent calculus for preferential conditional logic based on neighbourhood semantics. In Automated Reasoning with Analytic Tableaux and Related Methods (TABLEAUX2015), LNCS vol. 9323, H. de Nivelle, ed, pp. 115-134. Springer, 2015.

[15] S. Negri and J. von Plato. Structural Proof Theory, Cambridge University Press, 2001.

[16] S. Negri and J. von Plato. Proof Analysis, Cambridge University Press, 2011.

[17] E. Orlandelli. Proof analysis in deontic logics. In Deontic Logic and Normative Systems (DEON2014), LNAI, vol. 8554, F. Cariani et al., eds, pp. 139-148. Springer, 2014.

[18] M. Pauly. A modal logic for coalitional power in games. Journal of Logic and Computation, 12, 149-166, 2002

[19] M. Vardi. On epistemic logic and logical omniscience. In Theoretical Aspects of Reasoning about Knowledge. Proceedings of the 1986 Conference, Y. Halpern, ed., pp. 293-305. Morgan Kaufmann, 1986.

[20] A. Stolpe. QMML: quantified minimal modal logic and its application. Logic Journal of the $I G P L, \mathbf{1 1}, 557-575,2003$.

[21] G. Waagbø. Quantified modal logic with neighbourhood semantics. Zeitschrift für Mathematische Logik und Grundlagen der Mathematik, 38, 491-499, 1992.

Received Day Month year. 